\title{
Prognostic Utility of Platelet-Lymphocyte Ratio, Neutrophil-Lymphocyte Ratio and Monocyte-Lymphocyte Ratio in Head and Neck Cancers: A Detailed PRISMA Compliant Systematic Review and Meta-Analysis
}

\author{
Chellan Kumarasamy ${ }^{1}$, Vaibhav Tiwary ${ }^{2}\left(\mathbb{D}\right.$, Krishnan Sunil $^{3}$, Deepa Suresh ${ }^{4}$, Sameep Shetty ${ }^{5}$, \\ Gothandam Kodiveri Muthukaliannan ${ }^{2}$ D, Siddhartha Baxi ${ }^{6}$ and Rama Jayaraj ${ }^{7, *(D)}$
}

check for updates

Citation: Kumarasamy, C.; Tiwary, V.; Sunil, K.; Suresh, D.; Shetty, S.; Muthukaliannan, G.K.; Baxi, S.; Jayaraj, R. Prognostic Utility of Platelet-Lymphocyte Ratio, Neutrophil-Lymphocyte Ratio and Monocyte-Lymphocyte Ratio in Head and Neck Cancers: A Detailed PRISMA Compliant Systematic Review and Meta-Analysis. Cancers 2021, 13, 4166. https://doi.org/ $10.3390 /$ cancers13164166

Academic Editor: Amanda Psyrri

Received: 13 June 2021

Accepted: 16 August 2021

Published: 19 August 2021

Publisher's Note: MDPI stays neutral with regard to jurisdictional claims in published maps and institutional affiliations.

Copyright: (c) 2021 by the authors. Licensee MDPI, Basel, Switzerland. This article is an open access article distributed under the terms and conditions of the Creative Commons Attribution (CC BY) license (https:// creativecommons.org/licenses/by/ $4.0 /)$.
1 School of Health and Medical Sciences, Curtin University, Perth, WA 6102, Australia; chellank54@gmail.com

2 School of Biosciences and Technology, Vellore Institute of Technology (VIT), Vellore 632014, India; vaibhavtiwary1397@gmail.com (V.T.); gothandam@gmail.com (G.K.M.)

3 Department of Radiation Oncology, Mayo Clinic Florida, Jacksonville, FL 32224, USA; Krishnan.Sunil@mayo.edu

4 Division of Endocrinology, Department of Internal Medicine, Mayo Clinic Florida, Jacksonville, FL 32224, USA; Deepa.Suresh@mayo.edu

5 Department of Oral and Maxillofacial Surgery, Manipal College of Dental Sciences, Mangalore, Manipal Academy of Higher Education, A Constituent of MAHE, Manipal 576104, India; sameep.shetty@manipal.edu

6 Radiation Oncology, Genesiscare Gold Coast, John Flynn Hospital, 42 Inland Drive, Tugun, QLD 4224, Australia; Siddhartha.baxi@genesiscare.com

7 Northern Territory Institute of Research and Training, Darwin, NT 0909, Australia

* Correspondence: jramamoorthi@gmail.com

Simple Summary: Inflammation plays a major role in cancer development and progression and has the potential to be used as a prognostic marker in cancer. Previous studies have attempted to evaluate PLR, NLR and MLR as indicators of inflammation/prognostic markers in cancer, but there is no common consensus on its application in clinical practice. The aim of this systematic review and meta-analysis (a) assess the prognostic efficacy of all three prognostic markers in comparison to each other and, (b) investigate the prognostic potential of these three markers in HNC. The study followed PRISMA guidelines, with literature being collated from multiple bibliographic databases. Preliminary and secondary screening were carried out using stringent inclusion/exclusion criteria.

Abstract: Inflammation plays a major role in cancer development and progression and has the potential to be used as a prognostic marker in cancer. Previous studies have attempted to evaluate Platelet-to-lymphocyte ratio (PLR), neutrophil-lymphocyte ratio (NLR) or monocyte-lymphocyte ratio (MLR) as indicators of inflammation/prognostic markers in cancer, but there is no common consensus on their application in clinical practice. The aim of this systematic review and meta-analysis is to (a) assess the prognostic efficacy of all three prognostic markers in comparison to each other and (b) investigate the prognostic potential of these three markers in HNC. The study followed PRISMA guidelines, with the literature being collated from multiple bibliographic databases. Preliminary and secondary screening were carried out using stringent inclusion/exclusion criteria. Meta-analysis was carried out on selected studies using CMA software and HR as the pooled effect size metric. A total of 49 studies were included in the study. The pooled HR values of PLR, NLR and MLR indicated that they were significantly correlated with poorer OS. The pooled effect estimates for PLR, NLR and MLR were 1.461 (95\% CI 1.329-1.674), 1.639 (95\% CI 1.429-1.880) and 1.002 (95\% CI 0.720-1.396), respectively. Significant between-study heterogeneity was observed in the meta-analysis of all three. The results of this study suggest that PLR, NLR and MLR ratios can be powerful prognostic markers in head and neck cancers that can guide treatment. Further evidence from large-scale clinical studies on patient cohorts are required before they can be incorporated as a part of the clinical method. PROSPERO Registration ID: CRD42019121008 
Keywords: PLR; NLR; MLR; HNC; prognosis; meta-analysis; systematic review

\section{Introduction}

It is an established fact that cancer pathophysiology relies heavily on the manipulation of the immune system involved in cancer cell growth, proliferation and tumorigenesis [1]. Immunological involvement, or, more specifically, inflammation, has been defined as one of the hallmarks of cancer and plays a role in malignancy, angiogenesis and genomic instability [2]. Studies have shown that the presence of inflammation and inflammatory markers in cancer is associated with a change in prognosis [3]. Therefore, an assessment of the magnitude of inflammation in cancer patients could be used to determine disease prognosis. The magnitude of inflammation could be indirectly explored via the measurement of malnutrition and systemic inflammation-based indicators such as neutrophil-lymphocyte ratio (NLR), platelet-lymphocyte ratio (PLR) and monocyte-lymphocyte ratio (MLR). NLR can act as a proxy measurement of the degree of inflammation in cancers, as inflammation leads to systemic alterations in the levels of peripheral blood leukocytes [4,5]. Similarly, platelets release pro-inflammatory mediators, such as cytokines and chemokines, which intensify the inflammatory microenvironment in tumors, resulting in PLR being another viable measure of inflammation [6,7]. In addition, monocytes also have a key role in inflammation, as seen in their presence in atherosclerosis [8]. Individually, or in combination, these measures could be used as prognostic markers in cancer. The dense immune cell influx may explain the robust immune response in HPV-driven head and neck cancers and its potential to eliminate some of the tumor cells. An assessment of the prognosis of patients with cancer is significant, since a precise prediction of prognosis will permit appropriate treatment, improving patient outcomes [9]. The systemic inflammatory response elicited by the immune cells seen in cancer is interconnected with the nutritional depletion seen in cancer patients and affects the prognosis and course of the disease independently of tumor stage [10]. The host immune system is involved in cancer initiation, progression and metastasis. In addition, the immune-inflammatory retort to the toxic chemoradiation therapy adds to its clinical benefits. Lymphocytes represent one third of the total white blood cells, play a significant role in cellular immunity and may stimulate the clearance of malignant cells [11]. Antagonizing the action of lymphocytes, the monocytes enable tumor progression by promoting angiogenesis and immunosuppression [12]. An assessment of the MLR at different stages pretreatment or post-treatment can help in anticipating the prognosis of the disease and improving treatment decisions [13].

The relative ease of assessment, swift detection and minimal cost using NLR, PLR and MLR offers a lucrative option to gauge cancer prognosis and guide treatment. Furthermore, the need for only peripheral blood samples with little to no patient discomfort or pain increases the patient compliance [14]. Current methods for cancer prognosis in patients involve the use of molecular markers (such as BRCA1 in breast cancer and EGFR in NSCLC) $[15,16]$, which require complex and expensive assays for measurement and quantification (immunohistochemistry, q-RT PCR) [17,18], while also generating a greater degree of patient discomfort (biopsy), with an additional risk of seeding of tumor cells. Interpretation of tumor markers with complex assays does offer vital information on cancer prognosis and tumor-specific immunotherapy but they are not ideal for the constant and real-time monitoring of prognosis, as they are expensive, cumbersome and time-consuming. Therefore, significant research interest has been directed towards the use of PLR, NLR and MLR as additional or supplementary biomarkers for cancer prognosis.

Clinical studies have further explored the use of PLR, NLR and MLR as pre-operative prognostic biomarkers in addition to assessing their utility as diagnostic cancer biomarkers $[19,20]$. Further, the vast literature on these serum biomarkers has provided an impetus to conduct systematic review and meta-analysis studies [21,22]. However, a knowledge gap that exists, despite the presence of previous reviews, is that no systematic review or 
meta-analysis study published to date has investigated either (a) the prognostic efficacy of all three prognostic markers, PLR, NLR and MLR, in comparison to each other, or (b) the comparative utility of these markers with regard to HNC cancers. Previous systematic review and meta-analysis studies have either only focused on a single cancer type, with no study focusing on assessing the effectiveness of all three proposed biomarkers in $\mathrm{HNC}$, and / or comparing and contrasting all three biomarkers against each other. While Mellor et al. recently published a systematic review and meta-analysis study, including all cancer types, the study only focused on NLR alone as the prognostic marker of choice [23]. Similarly, Zhu et al. focused on multiple inflammatory markers, with emphasis on PLR and NLR, but limited their study to ovarian cancer [24], while another study by Zhang et al. limited their analysis to only colorectal cancer [21].

This comprehensive systematic review and meta-analysis study seeks to amend the aforementioned knowledge gap and attempts to provide a better understanding of the utility of PLR, NLR and MLR as prognostic markers in cancer. In addition, it seeks to highlight the comparative efficacy of PLR, NLR and MLR in each studied cancer type.

\section{Methods}

\subsection{Search Strategy}

This systematic review and meta-analysis study was conducted based on the standard review guidelines and methodology as detailed in the Preferred Reporting Items for Systematic Review and Meta-Analyses Protocol (PRISMA-P) guidelines [25,26]. The PRISMA compliance has been delineated in the PRISMA checklist table provided in Supplementary Data Table S1. The search strategy was expansive and exhaustive, with multiple bibliographic databases being searched. These databases included EMBASE, MEDLINE, Science Direct, Scopus and Web of Science. These databases were used to scope out relevant studies published within a ten-year span of time, i.e., from July 1999 to July 2019. This time constraint was placed to the search strategy in order to keep the results of the search relevant to the current developments in cancer prognosis and treatment. The search strategy also allowed for scoping of the reference lists of review articles and other publications for additional relevant studies for inclusion in the review. The bibliographic search was conducted via the construction of a logic grid and subsequent identification of specific 'keywords'. These 'keywords' were then used to construct 'search strings', which allowed for a thorough and robust search of the bibliographic databases.

Keywords: Platelet-to-Lymphocyte Ratio (PLR), Neutrophil-to-Lymphocyte Ratio (NLR), Monocyte-to-Lymphocyte Ratio (MLR), Biomarker, Head and Neck Cancer, Prognosis, Survival (Overall Survival, Disease Free Survival, Disease Specific Survival), Patient Study, Cohort, Blood, Systematic-review, Meta-analysis.

The search was conducted individually by two independent reviewers (CK and RJ), in order to eliminate the likelihood of selection bias occurring. Primary article and study screening was based on the pertinence of the title and abstract of each publication to the topic of the systematic review and meta-analysis being undertaken. The screening was conducted simultaneously alongside the initial search, under the discretion of the two reviewers (CK and RJ). Additional studies from the reference lists of reviews and other included publications were screened for and included after primary screening. Any disputes and differences in opinion arising during initial screening were settled through the inclusion of the third reviewer (VT).

\subsection{Study Selection}

After the primary screening procedure, the full texts of articles were subject to selection based on specific, predefined inclusion and exclusion criteria (secondary screening). The inclusion and exclusion criteria were based on previous similar systematic review and meta-analysis studies, adapted to the parameters of this study. 


\subsection{Inclusion Criteria}

1. The studies must discuss the survival outcome of HNC cancer patients based on PLR, NLR and MLR levels.

2. The survival outcome must be presented in the form of HR (hazard ratios) and 95\% CI (confidence intervals).

3. The survival outcome must be presented in the form of Kaplan-Meier curves, along with patient cohort information, for each treatment arm represented in the KM Curves. (This is required only if the HR and 95\% CI values have not been presented in the manuscript, as the above information is required to extract approximated HR values.)

\subsection{Exclusion Criteria}

1. Conference abstracts, reviews, letters to the editor and other non-clinical literature will not be considered for either systematic review or meta-analysis.

2. Included studies must be clinical studies or involve patient samples. (In vitro, in silico and animal studies will be excluded.)

3. Unpublished or non-peer-reviewed literature will be excluded.

4. Studies that do not focus on survival outcomes and prognosis aspects of PLR, NLR and MLR in cancer patients will not be considered.

5. If the sample size of each individual study is of low power (sample size $<10$ ), they will be excluded.

No limitations were placed on the types of patients involved, their clinicopathological characteristics or their demographic characteristics. No restrictions were placed based on age, sex, ethnicity, location, follow-up period, duration of treatment or method of treatment.

\subsection{Data Extraction and Recording}

After the secondary screening procedure, based on the predefined inclusion and exclusion criteria, the full-text formats of the selected studies were collated and subjected to a data extraction process. Data extraction followed a top-down approach, with the selected full-text studies being examined for relevant patient and study data individually by three reviewers, a process designed to generate redundancy, while reducing individual error. A standardized data extraction form, containing all the required data items, was prepared using Microsoft Excel and utilized by the reviewers to extract the data. After individual data extraction was performed by all reviewers, duplicated information/studies were removed. The resulting dataset of study information was collated into a single database for further analysis. The data items extracted from full-text versions of individual studies included:

1. Author names;

2. Year of publication;

3. Marker studied (PLR, NLR or MLR);

4. Size of patient cohort;

5. Diagnostic methods;

6. Follow-up period;

7. Gender split of cohort;

8. TNM staging split of cohort;

9. Survival endpoint of each study (overall survival, disease-free survival, diseasespecific survival);

10. General features of each study (will be presented as short qualitative opinions/observations of reviewers, for each study being included).

\subsection{Quality Assessment}

The quality assessment of the studies was based on the standard Newcastle-Ottawa Scale (NOS) for the quality assessment of nonrandomized studies in meta-analysis [27]. This scale presents a 'star system', which assigns each of the 3 broad parameters of the study (1-4 stars range) in increasing order of quality. The 3 broad perspectives being 
assessed include the selection of study groups in each individual study, the comparability of groups and the ascertainment of exposure/outcome of interest for case-control/cohort studies [28-32].

\subsection{Meta-Analysis}

The meta-analysis was conducted using the Comprehensive Meta-Analysis (CMA) software (version 3.3.070; Biostat, Englewood, NJ, USA) [33]. The hazard ratio was chosen as the appropriate effect size metric for this study, and overall survival (OS) was selected as the survival endpoint for the meta-analysis. The HR indicates the probability of survival of the patient cohort in each included study and was pooled across all included studies to determine the likelihood of overall survival (OS) of patients, across all studies. The pooled results were represented visually using forest plots. Meta-analyses on PLR, NLR and MLR were performed independently, to determine the prognostic effectiveness of each, as a cancer marker. Statistical significance ( $p$-values) of each of the three aforementioned prognostic markers was also calculated. The meta-analysis was conducted based on the random-effects model to account for inherent heterogeneity between each of the included studies [34].

\subsection{Assessment of Heterogeneity}

Assessment of heterogeneity was conducted using 3 parameters in order to increase the robustness of analysis [35-39]. The Higgins $I^{2}$ statistic was used as the primary method to determine heterogeneity, as it has a high power of detection of heterogeneity [40]. However, as $\mathrm{I}^{2}$ is not an infallible metric of heterogeneity, and can provide biased results in small meta-analyses [41], we also used the Cochran' $\mathrm{Q}$ and Tau ${ }^{2}$ parameters to assess heterogeneity alongside the $\mathrm{I}^{2}$ statistic $[42,43]$.

\subsection{Subgroup Analysis}

The major meta-analysis subgroups selected for this study were cohorts of studies that assessed PLR, NLR and MLR. As sufficient data were not available, only the survival endpoint of OS was assessed. Additionally, no subgroup analysis was carried out based on the demographic or clinicopathological characteristics, due to a lack of sufficient high-quality studies with comparable data, which would lead to a lack of power in the subsequent statistical analysis.

\subsection{Publication Bias}

Publication bias assessment was conducted as per PRISMA guidelines. The Egger's graphical bias indicator test was used to construct a funnel plot [44]. The funnel plot symmetry along the regression line was used to assess the existence of publication bias, wherein the symmetry of the funnel plot inversely correlated to the degree of publication bias in the meta-analysis study. Adjustment for small and missing studies was carried out via imputation of possible small studies using the Orwin's Fail-Safe N test [45]. The Begg and Mazumdar's Rank Correlation test was used to check correlation between ranks of effect sizes and variances [46], wherein a positive result indicated accurate publication bias assessment.

\section{Results}

The search strategy yielded a total of 28,716 studies across all databases. The majority of these studies were screened out by reviewers during the initial primary screening process, due to a lack of relevance to the topic of review. After primary screening, full texts of 120 articles were obtained, which were then further assessed for duplicates (which were removed) and adherence to pre-defined inclusion and exclusion criteria. This secondary screening process eliminated 71 studies, which left 49 studies suitable for inclusion in the systematic review and meta-analysis. However, due to a lack of sufficient quality data in all screened studies, only 34 publications that had the appropriate effect size data (HR and 
95\% CI values for OS) were included in the final meta-analysis. The data were subsequently extracted from the selected studies as per the defined data extraction procedure and used to construct a systematic review table and perform the meta-analysis. The entire process was monitored by the second and third reviewer (RJ and VT) at all stages. The selection process is delineated in Figure 1.

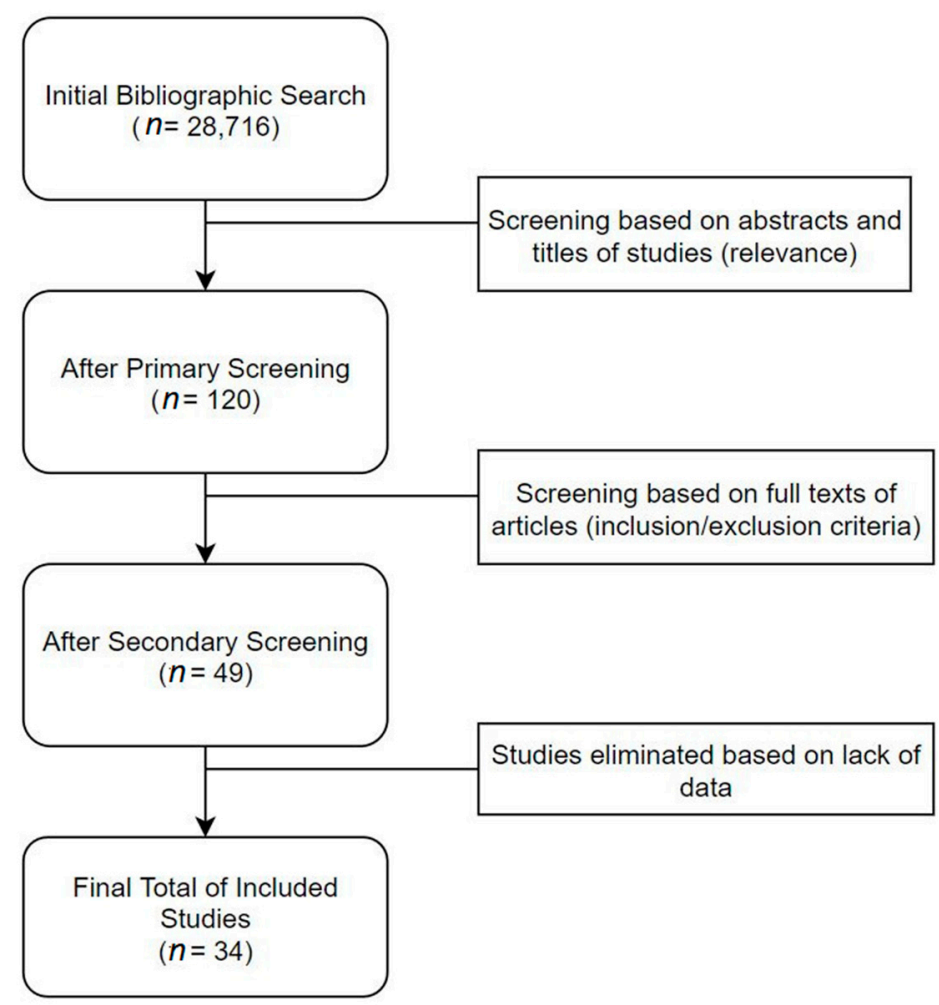

Figure 1. Search Strategy.

Study Characteristics: The 49 studies included in this systematic review and metaanalysis study were conducted across nine countries across the globe, as described in Table 1. The largest number of published studies were from China $(n=28)$. Other countries that featured in multiple publications on this topic included Japan $(n=9)$, the United Kingdom $(n=3)$, Taiwan $(n=3)$ and the Republic of Korea $(n=2)$, while Italy $(n=1)$, Switzerland $(n=1)$, Thailand $(n=1)$ and Turkey $(n=1)$ each only featured in a single publication. 
Table 1. Study characteristics of included studies for systematic review.

\begin{tabular}{|c|c|c|c|c|c|c|c|c|c|c|c|}
\hline Author Name & $\begin{array}{c}\text { Year of } \\
\text { Publication }\end{array}$ & $\begin{array}{c}\text { Prognostic } \\
\text { Parameter } \\
\text { (PLR/NLR/MLR) }\end{array}$ & Cohort Size & $\begin{array}{l}\text { Anatomic Location of } \\
\text { Cancer }\end{array}$ & $\begin{array}{l}\text { Country of } \\
\text { Study }\end{array}$ & Type of Study & Gender & Stage of Cancer & Metastasis & $\begin{array}{l}\text { Risk } \\
\text { Factors }\end{array}$ & Age \\
\hline Wen et al. [47] & 2018 & $\begin{array}{l}\text { PLR } \\
\text { NLR }\end{array}$ & 723 & $\begin{array}{l}\text { Esophageal }(65 \%) \\
\text { Gastric }(35 \%)\end{array}$ & UK & $\begin{array}{l}\text { retrospective } \\
\text { study }\end{array}$ & $\begin{array}{l}\text { male }(75.5 \%) \\
\text { female }(24.5 \%)\end{array}$ & $\begin{array}{l}\text { T0 }(4.6 \%) \\
\text { T1 }(14.4 \%) \\
\text { T2 }(21.0 \%) \\
\text { T3 }(51.9 \%) \\
\text { T4 }(8.2 \%)\end{array}$ & M1 2.5\% & NA & $66.1 \pm 10.5$ \\
\hline Kawakita et al. [48] & 2016 & $\begin{array}{l}\text { PLR } \\
\text { NLR }\end{array}$ & 140 & $\begin{array}{c}\text { Parotid gland }(78 \%) \\
\text { Submandibular gland } \\
(20 \%) \\
\text { Others }(2 \%)\end{array}$ & Japan & $\begin{array}{l}\text { retrospective } \\
\text { study }\end{array}$ & $\begin{array}{c}\text { male }(86 \%) \\
\text { female }(14 \%)\end{array}$ & $\begin{array}{c}\text { T1 }(9 \%) \\
\text { T2 }(26 \%) \\
\text { T3 }(20 \%) \\
4 a(44 \%) \\
4 \mathrm{~b}(1 \%)\end{array}$ & $\begin{array}{l}\text { M0 }(93 \%) \\
\text { M1 }(7 \%)\end{array}$ & NA & $64(26-84)$ \\
\hline Chen et al. [49] & 2014 & $\begin{array}{l}\text { NLR } \\
\text { PLR }\end{array}$ & 211 & Nasopharynx (100\%) & China & $\begin{array}{l}\text { retrospective } \\
\text { study }\end{array}$ & $\begin{array}{c}\text { male }(85.8 \%) \\
\text { female }(14.2 \%)\end{array}$ & NA & NA & NA & $46(14-72)$ \\
\hline Zhang et al. [50] & 2015 & $\begin{array}{l}\text { NLR } \\
\text { PLR }\end{array}$ & 468 & Esophagus $(100 \%)$ & China & $\begin{array}{l}\text { retrospective } \\
\text { study }\end{array}$ & $\begin{array}{c}\text { male }(80.3 \%) \\
\text { female }(19.7 \%)\end{array}$ & $\begin{array}{c}\text { stage I }(9.8 \%) \\
\text { stage II }(42.6 \%) \\
\text { stage III }(47.6 \%)\end{array}$ & NA & $\begin{array}{l}\text { Smoking } \\
\text { Alcohol con- } \\
\text { sumption }\end{array}$ & $59.5 \pm 9.0$ \\
\hline Jiang et al. [51] & 2017 & $\begin{array}{l}\text { PLR } \\
\text { NLR } \\
\text { LMR }\end{array}$ & 78 & Thyroid (100\%) & China & $\begin{array}{l}\text { retrospective } \\
\text { study }\end{array}$ & $\begin{array}{l}\text { male }(43.6 \%) \\
\text { female }(56.4 \%)\end{array}$ & $\begin{array}{l}\text { stage I }(23.1 \%) \\
\text { stage II }(20.5 \%) \\
\text { stage III }(20.5 \%) \\
\text { stage IV }(35.9 \%)\end{array}$ & M1 (2.6\%) & NA & $47.3 \pm 13.8$ \\
\hline Li et al. [52] & 2016 & $\begin{array}{l}\text { PLR } \\
\text { NLR } \\
\text { LMR }\end{array}$ & 388 & Nasopharynx $(100 \%)$ & China & $\begin{array}{l}\text { retrospective } \\
\text { study }\end{array}$ & NA & NA & M0 (100\%) & NA & \\
\hline Feng et al. [53] & 2014 & $\begin{array}{l}\text { NLR } \\
\text { PLR }\end{array}$ & 483 & Esophagus (100\%) & China & $\begin{array}{l}\text { retrospective } \\
\text { study }\end{array}$ & $\begin{array}{l}\text { male }(85.1 \%) \\
\text { female }(14.9 \%)\end{array}$ & $\begin{array}{l}\text { T1 }(18.0 \%) \\
\text { T2 }(16.6 \%) \\
\text { T3 }(54.9 \%) \\
\text { T4 }(10.5 \%)\end{array}$ & $\begin{array}{l}\text { M0 (56.7\%) } \\
\text { M1 (43.3\%) }\end{array}$ & NA & $59.1 \pm 8.0$ \\
\hline Jiang et al. [54] & 2015 & PLR & 1261 & Nasopharynx (100\%) & China & $\begin{array}{l}\text { retrospective } \\
\text { study }\end{array}$ & $\begin{array}{c}\text { male }(72.9 \%) \\
\text { female }(27.1 \%)\end{array}$ & $\begin{array}{c}\text { Clinical stage } \\
\text { I (3.25) } \\
\text { II (14.8\%) } \\
\text { III (52.7\%) } \\
\text { IV (29.4\%) } \\
\text { Tumor stage } \\
\text { T1 }(8.6 \%) \\
\text { T2 }(23.6 \%) \\
\text { T3 }(46.2 \%) \\
\text { T4 (21.5\%) } \\
\text { Node stage } \\
\text { N0 (16.8\%) } \\
\text { N1 (36.6\%) } \\
\text { N2 (37.7\%) } \\
\text { N3 }(9.0 \%)\end{array}$ & $\begin{array}{l}\text { M1 (12.4) } \\
\text { M0 (87.6) }\end{array}$ & $\begin{array}{l}\text { Smoking } \\
\text { Chronic } \\
\text { HBV } \\
\text { infection } \\
\text { Cardiovascular } \\
\text { disease } \\
\text { Diabetes } \\
\text { mellitus } \\
\text { Family } \\
\text { history of } \\
\text { NPC }\end{array}$ & $46(39-55)$ \\
\hline
\end{tabular}


Table 1. Cont.

\begin{tabular}{|c|c|c|c|c|c|c|c|c|c|c|c|}
\hline Author Name & $\begin{array}{c}\text { Year of } \\
\text { Publication }\end{array}$ & $\begin{array}{c}\text { Prognostic } \\
\text { Parameter } \\
\text { (PLR/NLR/MLR) }\end{array}$ & Cohort Size & $\begin{array}{l}\text { Anatomic Location of } \\
\text { Cancer }\end{array}$ & $\begin{array}{l}\text { Country of } \\
\text { Study }\end{array}$ & Type of Study & Gender & Stage of Cancer & Metastasis & $\begin{array}{l}\text { Risk } \\
\text { Factors }\end{array}$ & Age \\
\hline Jung et al. [55] & 2015 & $\begin{array}{l}\text { NLR } \\
\text { PLR }\end{array}$ & 119 & Esophagus (100\%) & Korea & $\begin{array}{l}\text { retrospective } \\
\text { study }\end{array}$ & $\begin{array}{l}\text { male }(94.1 \%) \\
\text { female }(5.9 \%)\end{array}$ & $\begin{array}{c}\text { Pathological stage } \\
\text { I }(31.1 \%) \\
\text { II } 33(27.7 \%) \\
\text { III } 49(41.2)\end{array}$ & M0 (100\%) & NA & $63.64 \pm 8.42$ \\
\hline He et al. [56] & 2016 & $\begin{array}{l}\text { NLR } \\
\text { PLR }\end{array}$ & 317 & Esophagus (100\%) & China & $\begin{array}{l}\text { retrospective } \\
\text { study }\end{array}$ & $\begin{array}{l}\text { male }(84.5 \%) \\
\text { female }(15.5 \%)\end{array}$ & $\begin{array}{c}\text { TNM } \\
\text { I-II } 68.5 \%) \\
\text { III-IV }(31.5 \%) \\
\end{array}$ & $\begin{array}{l}\text { M0 (53.9\%) } \\
\text { M1 (46.1\%) }\end{array}$ & $\begin{array}{l}\text { Smoking } \\
\text { Alcohol } \\
\text { consumption }\end{array}$ & $60(37-77)$ \\
\hline Jiang et al. [57] & 2016 & $\begin{array}{l}\text { NLR } \\
\text { PLR }\end{array}$ & 70 & Thyroid (100\%) & China & $\begin{array}{l}\text { retrospective } \\
\text { study }\end{array}$ & $\begin{array}{c}\text { male }(40 \%) \\
\text { female }(60 \%)\end{array}$ & $\begin{array}{l}\text { Stage III or IV } \\
(50.0 \%)\end{array}$ & M1 (2.9\%) & NA & $47.7 \pm 13.9$ \\
\hline Hirahara et al. [58] & 2017 & $\begin{array}{l}\text { LMR } \\
\text { NLR } \\
\text { PLR }\end{array}$ & 147 & Esophagus (100\%) & Japan & $\begin{array}{l}\text { retrospective } \\
\text { study }\end{array}$ & $\begin{array}{l}\text { male }(89.8 \%) \\
\text { female }(10.2 \%\end{array}$ & $\begin{array}{c}\text { Pathological stage } \\
\text { Ia-1b }(40.1 \%) \\
2 \mathrm{a}-2 \mathrm{~b}(22.4 \%) \\
\text { 3a-3c }(37.4 \%) \\
\end{array}$ & NA & NA & NA \\
\hline Ong et al. [59] & 2016 & $\begin{array}{l}\text { LMR } \\
\text { NLR } \\
\text { PLR }\end{array}$ & 133 & Tongue (100\%) & China & $\begin{array}{l}\text { retrospective } \\
\text { study }\end{array}$ & $\begin{array}{l}\text { male }(53.4 \%) \\
\text { female }(46.6 \%)\end{array}$ & $\begin{array}{c}\text { pT classification, } n \\
(\%) \\
\text { T1 }(39.1 \%) \\
\text { T2 }(60.9 \%)\end{array}$ & $\begin{array}{l}\text { M0 }(96.2 \%) \\
\text { M1 }(3.8 \%)\end{array}$ & NA & $51.92(24-74)$ \\
\hline Mao et al. [60] & 2017 & PLR & 899 & Larynx $(100 \%)$ & China & $\begin{array}{l}\text { retrospective } \\
\text { study }\end{array}$ & $\begin{array}{l}\text { male }(97.1 \%) \\
\text { female }(2.9 \%)\end{array}$ & $\begin{array}{c}\mathrm{T} \\
1(22.9 \%) \\
2(28.9 \%) \\
3(29.1 \%) \\
4(19.0 \%) \\
\mathrm{N} \\
0(81.2 \%) \\
1(9.7 \%) \\
2(8.6 \%) \\
3(0.6 \%)\end{array}$ & M0 (100\%) & $\begin{array}{l}\text { Smoking } \\
\text { Alcohol } \\
\text { consumption }\end{array}$ & $60(22-87)$ \\
\hline Dutta et al. [61] & 2011 & $\begin{array}{l}\text { NLR } \\
\text { PLR }\end{array}$ & 112 & Esophagus (100\%) & UK & $\begin{array}{l}\text { retrospective } \\
\text { study }\end{array}$ & $\begin{array}{c}\text { male }(75.9 \%) \\
\text { female }(24.1 \%)\end{array}$ & $\begin{array}{c}\text { TNM stage } \\
\text { I } 17.9 \% \\
\text { II } 34.8 \% \\
\text { III } 46.4 \% \\
\text { IV } 0.9 \%\end{array}$ & NA & NA & $\begin{array}{c}<65(60.7 \%) \\
65-74(33.9 \%) \\
\geq 75(5.4 \%)\end{array}$ \\
\hline Li et al. [62] & 2016 & $\begin{array}{l}\text { NLR } \\
\text { PLR }\end{array}$ & 409 & Nasopharynx (100\%) & China & $\begin{array}{l}\text { retrospective } \\
\text { study }\end{array}$ & $\begin{array}{l}\text { male }(70.4 \%) \\
\text { female }(29.6 \%)\end{array}$ & $\begin{array}{l}\text { (I-III) }(18.8 \%) \\
(\text { III-IV) }(81.2 \%)\end{array}$ & $\begin{array}{l}\text { M0 }(84.4 \%) \\
\text { M1 }(15.6 \%)\end{array}$ & NA & 45 (18-77) \\
\hline Messager et al. [63] & 2015 & PLR & 153 & Esophagus (100\%) & UK & $\begin{array}{l}\text { live patient } \\
\text { samples }\end{array}$ & $\begin{array}{c}\text { male }(83.7 \%) \\
\text { female }(16.3 \%)\end{array}$ & & & & \\
\hline Hirahara et al. [64] & 2016 & $\begin{array}{l}\text { LMR } \\
\text { NLR } \\
\text { PLR }\end{array}$ & 147 & Esophagus (100\%) & Japan & $\begin{array}{l}\text { retrospective } \\
\text { study }\end{array}$ & $\begin{array}{l}\text { male }(89.8 \%) \\
\text { female }(10.2 \%)\end{array}$ & $\begin{array}{c}\text { Pathological stage } \\
\text { Ia-lb } 40.1 \% \\
\text { Ila-lib } 22.5 \% \\
\text { IIIa-IIIc } 37.4 \%\end{array}$ & NA & NA & NA \\
\hline
\end{tabular}


Table 1. Cont.

\begin{tabular}{|c|c|c|c|c|c|c|c|c|c|c|c|}
\hline Author Name & $\begin{array}{c}\text { Year of } \\
\text { Publication }\end{array}$ & $\begin{array}{c}\text { Prognostic } \\
\text { Parameter } \\
\text { (PLR/NLR/MLR) }\end{array}$ & Cohort Size & $\begin{array}{l}\text { Anatomic Location of } \\
\text { Cancer }\end{array}$ & $\begin{array}{l}\text { Country of } \\
\text { Study }\end{array}$ & Type of Study & Gender & Stage of Cancer & Metastasis & $\begin{array}{l}\text { Risk } \\
\text { Factors }\end{array}$ & Age \\
\hline Moon et al. [65] & 2015 & $\begin{array}{l}\text { NLR } \\
\text { PLR }\end{array}$ & 153 & $\begin{array}{c}\text { Oropharynx } 33.3 \% \\
\text { Nasopharynx } 31.4 \% \\
\text { Larynx } 18.3 \% \\
\text { Hypopharynx } 17.0 \%\end{array}$ & Korea & $\begin{array}{l}\text { live patient } \\
\text { samples }\end{array}$ & $\begin{array}{l}\text { male }(84.3 \%) \\
\text { female }(15.7 \%)\end{array}$ & $\begin{array}{c}\text { Clinical TNM stage } \\
\text { T1/T2 17.6\%/36.6\% } \\
\text { T3/T4 14.4\%/3.1.4\% } \\
\text { N0/N1 21.6\%/20.9\% } \\
\text { N2/N3 49.7\%/7.8\% } \\
\text { Overall I/II } \\
\text { 4.6\%/17.0\% } \\
\text { Overall III/IV } \\
\text { 13.7\%/64.75\% } \\
\end{array}$ & M0 (100\%) & $\begin{array}{l}\text { Smoking } \\
\text { Alcohol con- } \\
\text { sumption }\end{array}$ & $57(16-78)$ \\
\hline $\begin{array}{c}\text { Turri-Zanoni et al. } \\
\text { [67] }\end{array}$ & 2016 & $\begin{array}{l}\text { NLR } \\
\text { PLR }\end{array}$ & 215 & Paranasal sinus $(100 \%)$ & Italy & $\begin{array}{l}\text { retrospective } \\
\text { study }\end{array}$ & $\begin{array}{c}\text { male }(34 \%) \\
\text { female }(66 \%)\end{array}$ & $\begin{array}{c}\text { pT classification } \\
\text { pT1 19\% } \\
\text { pT2 18\% } \\
\text { pT3 22\% } \\
\text { pT4a 16\% } \\
\text { pT4b 25\% } \\
\end{array}$ & M0 (100\%) & NA & $65(8-87)$ \\
\hline Xie et al. [68] & 2014 & $\begin{array}{l}\text { NLR } \\
\text { PLR }\end{array}$ & 317 & Esophagus (100\%) & China & $\begin{array}{l}\text { retrospective } \\
\text { study }\end{array}$ & $\begin{array}{c}\text { male }(77 \%) \\
\text { female }(23 \%)\end{array}$ & $\begin{array}{l}\text { Tumor stage } \\
\text { Stage I } 88.4 \\
\text { Stage II } 69.7 \\
\text { Stage III } 42.4\end{array}$ & M0 (100\%) & NA & $58.1 \pm 8.9$ \\
\hline Bojaxhiu et al. [69] & 2018 & $\begin{array}{l}\text { NLR } \\
\text { PLR }\end{array}$ & 186 & $\begin{array}{c}\text { Oral cavity }(28 \%) \\
\text { Oropharynx }(45 \%) \\
\text { Hypopharynx }(15 \%) \\
\text { Larynx }(13 \%)\end{array}$ & Switzerland & $\begin{array}{l}\text { retrospective } \\
\text { study }\end{array}$ & $\begin{array}{c}\text { male }(79 \%) \\
\text { female }(22 \%)\end{array}$ & $\begin{array}{c}\text { UICC stage, N (\%) } \\
\text { I }(3 \%) \\
\text { II }(6 \%) \\
\text { III }(24 \%) \\
\text { IV }(68 \%)\end{array}$ & M0 (100\%) & $\begin{array}{l}\text { Smoking } \\
\text { Alcohol con- } \\
\text { sumption }\end{array}$ & $61(41-88)$ \\
\hline Sun et al. [70] & 2017 & $\begin{array}{l}\text { NLR } \\
\text { PLR }\end{array}$ & 148 & Nasopharynx (100\%) & China & $\begin{array}{l}\text { retrospective } \\
\text { study }\end{array}$ & $\begin{array}{l}\text { male }(83.8 \%) \\
\text { female }(16.2 \%)\end{array}$ & & M0 (100\%) & Smoking & $45(24-72)$ \\
\hline Sun et al. [71] & 2015 & $\begin{array}{l}\text { NLR } \\
\text { PLR }\end{array}$ & 251 & Nasopharynx (100\%) & China & $\begin{array}{l}\text { retrospective } \\
\text { study }\end{array}$ & $\begin{array}{l}\text { male }(71.7 \%) \\
\text { female }(28.3 \%)\end{array}$ & $\begin{array}{c}\text { UICC / AJCC stage } \\
\text { I } 2.4 \% \\
\text { II } 15.9 \% \\
\text { III } 47.4 \% \\
\text { IV } 34.3 \%\end{array}$ & M0 (100\%) & NA & $46(15-76)$ \\
\hline $\begin{array}{l}\text { Tangthongkum } \\
\text { et al. [72] }\end{array}$ & 2017 & PLR & 274 & Oral $(100 \%)$ & Thailand & $\begin{array}{l}\text { retrospective } \\
\text { study }\end{array}$ & $\begin{array}{c}\text { males }(64.4 \%) \\
\text { females }(35.6 \%)\end{array}$ & NA & NA & NA & $60(21-92)$ \\
\hline Ozturk et al. [73] & 2016 & $\begin{array}{l}\text { NLR } \\
\text { PLR }\end{array}$ & 57 & Tongue $(100 \%)$ & Turkey & $\begin{array}{l}\text { retrospective } \\
\text { study }\end{array}$ & $\begin{array}{l}\text { male }(38.6 \%) \\
\text { female }(61.4 \%)\end{array}$ & $\begin{array}{l}\text { stage I }(64.9 \%) \\
\text { stage II }(35.1 \%)\end{array}$ & M0 (100\%) & NA & $57.8(23-88)$ \\
\hline $\begin{array}{c}\text { Toyokawa et al. } \\
{[74]}\end{array}$ & 2016 & $\begin{array}{l}\text { NLR } \\
\text { PLR }\end{array}$ & 185 & Esophagus (100\%) & Japan & $\begin{array}{l}\text { retrospective } \\
\text { study }\end{array}$ & $\begin{array}{c}\text { male }(82.2 \%) \\
\text { female }(17.8 \%)\end{array}$ & $\begin{array}{c}\text { Clinical TNM stage } \\
\text { I } 36.2 \% \\
\text { II } 42.2 \% \\
\text { III/IV } 21.6 \%\end{array}$ & M0 (100\%) & NA & $\begin{array}{l}<6551.4 \% \\
\geq 6548.6 \%\end{array}$ \\
\hline
\end{tabular}


Table 1. Cont.

\begin{tabular}{|c|c|c|c|c|c|c|c|c|c|c|c|}
\hline Author Name & $\begin{array}{c}\text { Year of } \\
\text { Publication }\end{array}$ & $\begin{array}{c}\text { Prognostic } \\
\text { Parameter } \\
\text { (PLR/NLR/MLR) }\end{array}$ & Cohort Size & $\begin{array}{l}\text { Anatomic Location of } \\
\text { Cancer }\end{array}$ & $\begin{array}{l}\text { Country of } \\
\text { Study }\end{array}$ & Type of Study & Gender & Stage of Cancer & Metastasis & $\begin{array}{l}\text { Risk } \\
\text { Factors }\end{array}$ & Age \\
\hline Urabe et al. [75] & 2017 & $\begin{array}{l}\text { LMR } \\
\text { NLR } \\
\text { PLR }\end{array}$ & 1363 & $\begin{array}{l}\text { Resectable Gastric and } \\
\text { Esophagogastric } \\
\text { Junction }\end{array}$ & Japan & $\begin{array}{l}\text { retrospective } \\
\text { study }\end{array}$ & $\begin{array}{c}\text { male }(71.5 \%) \\
\text { female }(28.5 \%)\end{array}$ & $\begin{array}{c}\text { T stage } \\
\text { T1 } 58 \% \\
\text { T2 } 11.8 \% \\
\text { T3 } 17.6 \% \\
\text { T4 } 12.6 \%\end{array}$ & M0 (100\%) & NA & NA \\
\hline Wang et al. [76] & 2014 & PLR & 252 & $\begin{array}{l}\text { Upper aerodigestive } \\
\text { tract }\end{array}$ & China & $\begin{array}{l}\text { retrospective } \\
\text { study }\end{array}$ & $\begin{array}{c}\text { male }(68.7 \%) \\
\text { female }(31.3 \%)\end{array}$ & $\begin{array}{c}\text { Ann Arbor stage } \\
\text { IE } 61.5 \% \\
\text { IIE } 38.5 \%\end{array}$ & NA & NA & $41(9-80)$ \\
\hline Wei et al. [77] & 2015 & $\begin{array}{l}\text { NLR } \\
\text { PLR }\end{array}$ & 423 & Esophagus $(100 \%)$ & China & $\begin{array}{l}\text { retrospective } \\
\text { study }\end{array}$ & $\begin{array}{l}\text { male }(80.6 \%) \\
\text { female }(19.4)\end{array}$ & $\begin{array}{c}\text { TNM stage (AJCC, } \\
7 \text { th) } \\
\text { I }(12.8 \%) \\
\text { II }(39.7 \%) \\
\text { III }(33.6 \%) \\
\text { IV }(13.9 \%)\end{array}$ & $\begin{array}{l}\text { M0 (86.1) } \\
\text { M1 (13.9) }\end{array}$ & NA & $58(24-88)$ \\
\hline Xu et al. [78] & 2015 & $\begin{array}{l}\text { NLR } \\
\text { PLR }\end{array}$ & 468 & Esophagus $(100 \%)$ & China & $\begin{array}{l}\text { retrospective } \\
\text { study }\end{array}$ & $\begin{array}{c}\text { male }(88.9 \%) \\
\text { female }(11.1 \%)\end{array}$ & $\begin{array}{c}\text { Clinical stage } \\
\text { I } \\
\text { II } \\
\text { IIIA } \\
\text { IIIB + IIIC }\end{array}$ & NA & $\begin{array}{l}\text { Smoking } \\
\text { Alcohol } \\
\text { consumption }\end{array}$ & 58 \\
\hline Yang et al. [79] & 2018 & $\begin{array}{l}\text { NLR } \\
\text { PLR }\end{array}$ & 515 & Esophagus (100\%) & China & $\begin{array}{l}\text { retrospective } \\
\text { study }\end{array}$ & $\begin{array}{c}\text { male }(81.2 \%) \\
\text { female }(18.8 \%)\end{array}$ & $\begin{array}{c}\text { TNM Stage } \\
\text { I } \\
\text { II } \\
\text { III }\end{array}$ & M0 (100\%) & NA & 61(33-92) \\
\hline Ye et al. [80] & 2018 & $\begin{array}{l}\text { NLR } \\
\text { PLR }\end{array}$ & 427 & Nasopharynx (100\%) & China & $\begin{array}{l}\text { retrospective } \\
\text { study }\end{array}$ & $\begin{array}{c}\text { male }(71.9 \%) \\
\text { female }(28.1 \%)\end{array}$ & $\begin{array}{c}\text { TNM stage } \\
\text { I } 2.1 \\
\text { II } 18.7 \% \\
\text { III } 48.7 \% \\
\text { IV } 30.5 \%\end{array}$ & yes & NA & $48(17-82)$ \\
\hline Yuan et al. [81] & 2014 & $\begin{array}{l}\text { NLR } \\
\text { PLR }\end{array}$ & 327 & $\begin{array}{l}\text { Esophagogastric } \\
\text { junction }(100 \%)\end{array}$ & China & $\begin{array}{l}\text { retrospective } \\
\text { study }\end{array}$ & $\begin{array}{c}\text { male }(86.2 \%) \\
\text { female }(13.8 \%)\end{array}$ & $\begin{array}{c}\text { pTNM stage } \\
\text { I and II }(45.9 \%) \\
\text { III and IV }(54.1 \%)\end{array}$ & yes & NA & $\begin{array}{l}63.1 \pm 9.7 \\
(39-77)\end{array}$ \\
\hline Zhang et al. [82] & 2017 & $\begin{array}{l}\text { NLR } \\
\text { PLR }\end{array}$ & 355 & $\begin{array}{l}\text { Esophagogastric } \\
\text { junction }(100 \%)\end{array}$ & China & $\begin{array}{l}\text { retrospective } \\
\text { study }\end{array}$ & $\begin{array}{c}\text { male }(79.2 \%) \\
\text { female }(20.8 \%)\end{array}$ & $\begin{array}{l}\text { TNM stage } \\
\text { I, II (43.4\%) } \\
\text { III, IV (56.6\%) }\end{array}$ & NA & NA & $64(34-82)$ \\
\hline Chen et al. [20] & 2018 & $\begin{array}{l}\text { NLR } \\
\text { PLR } \\
\text { MLR }\end{array}$ & 361 & Larynx (100\%) & China & $\begin{array}{l}\text { retrospective } \\
\text { study }\end{array}$ & $\begin{array}{l}\text { male }(97.8 \%) \\
\text { female }(2.2 \%)\end{array}$ & $\begin{array}{c}\text { TNM stage } \\
\text { I } 31.6 \% \\
\text { II } 32.7 \% \\
\text { III } 19.7 \% \\
\text { IV } 16.0 \%\end{array}$ & yes & NA & $60(35-87)$ \\
\hline Hsu et al. [83] & 2009 & MLR & 1069 & Esophagus (100\%) & Taiwan & $\begin{array}{l}\text { retrospective } \\
\text { study }\end{array}$ & $\begin{array}{l}\text { male }(94.5 \%) \\
\text { female }(5.5 \%)\end{array}$ & $\begin{array}{c}\text { stage } \\
\text { I } 53(10.9 \%) \\
\text { II } 197(40.4 \%) \\
\text { III } 138(28.3 \%) \\
\text { IV } 100(20.5 \%)\end{array}$ & yes & NA & $63.8(34-88)$ \\
\hline
\end{tabular}


Table 1. Cont.

\begin{tabular}{|c|c|c|c|c|c|c|c|c|c|c|c|}
\hline Author Name & $\begin{array}{c}\text { Year of } \\
\text { Publication }\end{array}$ & $\begin{array}{c}\text { Prognostic } \\
\text { Parameter } \\
\text { (PLR/NLR/MLR) }\end{array}$ & Cohort Size & $\begin{array}{l}\text { Anatomic Location of } \\
\text { Cancer }\end{array}$ & $\begin{array}{l}\text { Country of } \\
\text { Study }\end{array}$ & Type of Study & Gender & Stage of Cancer & Metastasis & $\begin{array}{l}\text { Risk } \\
\text { Factors }\end{array}$ & Age \\
\hline Chien et al. [84] & 2016 & MLR & 2025 & Esophagus (100\%) & Taiwan & $\begin{array}{l}\text { retrospective } \\
\text { study }\end{array}$ & $\begin{array}{l}\text { male }(94.1 \%) \\
\text { female }(5.9 \%)\end{array}$ & $\begin{array}{c}\text { cStage T1-2N0 } \\
\text { T3-4N0 } \\
\text { T1-2N (+) } \\
\text { T3-4N (+) } \\
\text { Unknown }\end{array}$ & yes & NA & $55.2 \pm 9.8$ \\
\hline $\begin{array}{c}\text { Furukawa et al. } \\
\text { [85] }\end{array}$ & 2019 & LMR & 103 & Tongue $(100 \%)$ & Japan & $\begin{array}{l}\text { retrospective } \\
\text { study }\end{array}$ & $\begin{array}{c}\text { male }(53.8 \%) \\
\text { female }(46.2 \%)\end{array}$ & $\begin{array}{c}\text { stage } \\
\text { I, II } 84.5 \% \\
\text { III, IV } 15.5 \%\end{array}$ & yes & $\begin{array}{l}\text { Smoking } \\
\text { Alcohol con- } \\
\text { sumption }\end{array}$ & $63(26-92)$ \\
\hline Huang et al. [87] & 2015 & LMR & 348 & Esophagus (100\%) & China & $\begin{array}{l}\text { retrospective } \\
\text { study }\end{array}$ & $\begin{array}{c}\text { male }(87.1 \%) \\
\text { female }(12.9 \%)\end{array}$ & NA & yes & NA & $59.2 \pm 7.8$ \\
\hline Kano et al. [88] & 2016 & $\begin{array}{l}\text { NLR } \\
\text { PLR } \\
\text { LMR }\end{array}$ & 285 & $\begin{array}{c}\text { Larynx }(23.5 \%) \\
\text { Oropharynx }(40.7 \%) \\
\text { Hypopharynx }(35.8 \%)\end{array}$ & Japan & $\begin{array}{l}\text { retrospective } \\
\text { study }\end{array}$ & $\begin{array}{c}\text { male }(88.4 \%) \\
\text { female }(11.6 \%)\end{array}$ & $\begin{array}{c}\text { Clinical stage } \\
\text { II II (22.1\%) } \\
\text { III, IV }(77.9 \%) \\
\end{array}$ & no & NA & $61(37-80)$ \\
\hline Li et al. [89] & 2013 & LMR & 1547 & Nasopharynx (100\%) & China & $\begin{array}{l}\text { retrospective } \\
\text { study }\end{array}$ & $\begin{array}{c}\text { male } 72.7 \% \\
\text { female } 27.3 \%\end{array}$ & $\begin{array}{c}\text { Overall stage } \\
\text { IIII (21.6\%) } \\
\text { III-IV }(78.4 \%) \\
\end{array}$ & yes & NA & $51(6-87)$ \\
\hline Li et al. [52] & 2017 & $\begin{array}{l}\text { NLR } \\
\text { PLR } \\
\text { LMR }\end{array}$ & 249 & Nasopharynx (100\%) & China & $\begin{array}{l}\text { prospective } \\
\text { study }\end{array}$ & $\begin{array}{c}\text { male }(73.9 \%) \\
\text { female }(26.1 \%)\end{array}$ & $\begin{array}{c}\text { Clinical stage } \\
\text { I-II 26.1 } \\
\text { III-IV } 73.9 \\
\end{array}$ & yes & $\mathrm{NA}$ & $\begin{array}{l}\leq 50(65.9 \%) \\
>50(34.1 \%)\end{array}$ \\
\hline Liu et al. [90] & 2015 & $\begin{array}{l}\text { NLR } \\
\text { PLR } \\
\text { LMR }\end{array}$ & 326 & Esophagus (100\%) & China & $\begin{array}{l}\text { retrospective } \\
\text { study }\end{array}$ & $\begin{array}{c}\text { male }(86.8 \%) \\
\text { female }(13.2 \%)\end{array}$ & $\begin{array}{c}\text { T stage } \\
\text { T1 }(18.1 \%) \\
\text { T2 }(18.4 \%) \\
\text { T3 }(53.7 \%) \\
\text { T4 }(9.8 \%)\end{array}$ & NA & NA & $\begin{array}{c}59.2 \pm 7.9 \\
(38-80)\end{array}$ \\
\hline Oya et al. [91] & 2018 & $\begin{array}{l}\text { NLR } \\
\text { PLR } \\
\text { LMR }\end{array}$ & 441 & $\begin{array}{c}\text { Oral cavity } 44 \% \\
\text { Larynx } 28 \% \\
\text { Oropharynx 10\% } \\
\text { Hypopharynx 13\% } \\
\text { Other 5\% } \\
\end{array}$ & Japan & $\begin{array}{l}\text { retrospective } \\
\text { study }\end{array}$ & $\begin{array}{c}\text { male }(73 \%) \\
\text { female }(27 \%)\end{array}$ & $\begin{array}{l}\text { Stage } \\
\text { I } 32 \% \\
\text { II } 18 \% \\
\text { III } 15 \% \\
\text { IV } 35 \% \\
\end{array}$ & no & NA & $68(27-92)$ \\
\hline Yang et al. [92] & 2018 & $\begin{array}{l}\text { NLR } \\
\text { LMR }\end{array}$ & 197 & $\begin{array}{c}\text { Hypopharyngeal } \\
(100 \%)\end{array}$ & China & $\begin{array}{l}\text { retrospective } \\
\text { study }\end{array}$ & $\begin{array}{c}\text { male }(99.0 \%) \\
\text { female }(0.01 \%)\end{array}$ & $\begin{array}{c}\text { Clinical stage } \\
\text { I }(2.0 \%) \\
\text { II }(13.2 \%) \\
\text { III }(27.5 \%) \\
\text { IV }(57.4 \%)\end{array}$ & yes & $\begin{array}{l}\text { Smoking } \\
\text { Drinking }\end{array}$ & $\begin{array}{l}<59(50.8 \%) \\
\geq 59(49.2 \%)\end{array}$ \\
\hline
\end{tabular}


Most of these studies were large-scale retrospective studies, representing a total pooled patient cohort of 20,739 patients. The collated data across these 49 studies suggest that nearly all studies in this field of PLR-, NLR- and MLR-based cancer prognostics have focused on head and neck cancers (HNC), with the main anatomical locations described being the oral cavity, nasopharyngeal, oropharyngeal, hypopharyngeal, laryngealnasopharyngeal, laryngeal and tongue regions. Further observation of the demographic data also suggests that most studies included in this review involved a higher percentage of men, in comparison to women $(n=45)$, with only a few studies reporting a higher percentage of women in their studies $(n=4)$. Not all studies assessed all three prognostic indicators of PLR, NLR and MLR. A large proportion of the studies assessed PLR and NLR in conjunction $(n=24)$, with a smaller proportion assessing all three simultaneously $(n=13)$. A few studies were also noted to have assessed each of the prognostic markers individually, with PLR $(n=6)$ and MLR $(n=3)$ both being assessed equally, while no study assessed NLR individually $(n=0)$. While most studies did not present any information regarding underlying risk factors $(n=39)$, the studies that did provide this information highlighted smoking $(n=10)$ and drinking $(n=8)$ as the primary risk factors, while one study also detailed additional risk factors such as chronic HPV infection, CVD, diabetes and family history.

\section{Meta-Analysis}

As described in the methodology and protocol of the study, a meta-analysis of pooled HR and 95\% CI values was carried out under three subgroups, for the survival endpoint of OS. The subgroups analyzed were based on the three potential prognostic markers PLR, NLR and MLR. Each individual cohort of each study focusing uniquely on each of the three aforementioned prognostic markers was assessed as an independent study, contributing to the respective subgroup being analyzed. The pooled results were graphically represented in the form of forest plots.

\subsection{Meta-Analysis PLR Subgroup}

A total of 25 cohorts of studies were pooled for determining the prognostic impact of a change (increase) in PLR levels upon the overall survival of the patient cohort (Figure 2). Twenty-three studies showed a positive correlation between increased PLR levels and a worse disease outcome (poorer prognosis), out of which 18 studies showed a significant level of said correlation $(p<0.05)$. Interestingly, two other additional studies contradicted the rest, suggesting an inverse correlation between PLR levels and patient survival, thereby suggesting a better prognosis for OS. The pooled effect estimate (HR) was found to be statistically significant, at a value of $1.461(95 \%$ CI 1.329-1.674; $p=0.0001)$. Assessment of heterogeneity suggests that there is substantial heterogeneity between the studies included in the subgroup meta-analysis for PLR $\left(\mathrm{I}^{2}=80.320\right.$; $\mathrm{Tau}^{2}=0.050$; Cochran's $\left.Q=121.949\right)$.

\subsection{Meta-Analysis NLR Subgroup}

A total of 27 cohorts of studies were pooled for determining the prognostic impact of a change (increase) in NLR levels upon the overall survival of the patient cohort (Figure 3). The majority of the studies $(n=23)$ showed a positive correlation between increased NLR levels and a worse disease outcome (poorer prognosis), out of which 20 studies showed a significant level of said correlation $(p<0.05)$. Interestingly, only one other study contradicted the majority, suggesting an inverse correlation between NLR levels and patient survival, indicating a better prognosis for OS. The pooled effect estimate (HR) was found to be statistically significant, at a value of $1.639(95 \%$ CI $1.429-1.880 ; p=0.001)$. Assessment of heterogeneity suggests that there is substantial heterogeneity between the studies included in the subgroup meta-analysis for NLR $\left(\mathrm{I}^{2}=82.152\right.$; $\mathrm{Tau}^{2}=0.085$; Cochran's $\left.Q=145.674\right)$. 


\begin{tabular}{|c|c|c|c|c|c|c|c|}
\hline \multirow[t]{2}{*}{ Study name } & \multirow[b]{2}{*}{$\begin{array}{c}\text { Hazard } \\
\text { ratio }\end{array}$} & \multicolumn{3}{|c|}{ Statistics for each study } & \multirow[b]{2}{*}{$p$-Value } & \multirow[b]{2}{*}{$\begin{array}{r}\text { Relative } \\
\text { weight }\end{array}$} & \multirow[b]{2}{*}{$\begin{array}{c}\text { Std } \\
\text { Residual }\end{array}$} \\
\hline & & $\begin{array}{l}\text { Lower } \\
\text { limit }\end{array}$ & $\begin{array}{c}\text { Upper } \\
\text { limit }\end{array}$ & Z-Value & & & \\
\hline Hirahara et al.2016 (BMC Surg) & 0.500 & 0.253 & 0.990 & -1.989 & 0.047 & 2.03 & -2.67 \\
\hline Dutta et al.2011 & 0.940 & 0.599 & 1.476 & -0.269 & 0.788 & 3.38 & -1.47 \\
\hline Ong et al.2016 & 1.103 & 1.009 & 1.206 & 2.157 & 0.031 & 6.71 & -1.37 \\
\hline Xu et al.2015 & 1.120 & 0.874 & 1.436 & 0.894 & 0.371 & 5.28 & -1.15 \\
\hline Zhang et al.2017 & 1.124 & 0.789 & 1.601 & 0.647 & 0.517 & 4.22 & -1.01 \\
\hline Toyokawa et al. 2016 & 1.129 & 0.656 & 1.943 & 0.438 & 0.661 & 2.74 & -0.79 \\
\hline Urabe et al.2017 & 1.130 & 1.081 & 1.181 & 5.410 & 0.000 & 6.92 & -1.28 \\
\hline Zhang et al.2015 & 1.288 & 1.003 & 1.653 & 1.986 & 0.047 & 5.27 & -0.59 \\
\hline Chen et al.2014 & 1.311 & 1.086 & 1.582 & 2.825 & 0.005 & 5.90 & -0.55 \\
\hline Wen et al.2018 & 1.330 & 1.084 & 1.631 & 2.736 & 0.006 & 5.73 & -0.48 \\
\hline Yuan et al.2014 & 1.398 & 0.872 & 2.241 & 1.391 & 0.164 & 3.22 & -0.20 \\
\hline Oya et al.2018 & 1.490 & 1.039 & 2.138 & 2.165 & 0.030 & 4.15 & -0.00 \\
\hline Yang et al.2018 & 1.569 & 1.258 & 1.956 & 4.003 & 0.000 & 5.57 & 0.21 \\
\hline He et al.2018 & 1.595 & 1.172 & 2.170 & 2.971 & 0.003 & 4.67 & 0.25 \\
\hline Hirahara et al.2016 (Eur J Surg Onc) & 1.663 & 1.003 & 2.758 & 1.971 & 0.049 & 2.98 & 0.32 \\
\hline Li et al.2016 & 1.865 & 1.067 & 3.261 & 2.186 & 0.029 & 2.65 & 0.63 \\
\hline Hirahara et al.2017 & 1.886 & 1.043 & 3.411 & 2.099 & 0.036 & 2.46 & 0.63 \\
\hline Wang et al.2014 & 1.970 & 1.240 & 3.130 & 2.870 & 0.004 & 3.29 & 0.87 \\
\hline Kawakita et al.2016 & 1.980 & 1.153 & 3.400 & 2.477 & 0.013 & 2.76 & 0.81 \\
\hline Tangthongkum et al.2017 & 2.230 & 1.572 & 3.164 & 4.494 & 0.000 & 4.26 & 1.44 \\
\hline Feng et al.2014 & 2.245 & 1.735 & 2.904 & 6.154 & 0.000 & 5.19 & 1.63 \\
\hline Jung et al.2015 & 2.245 & 1.735 & 2.904 & 6.154 & 0.000 & 5.19 & 1.63 \\
\hline Sun et al.2017 & 2.450 & 1.407 & 4.267 & 3.166 & 0.002 & 2.67 & 1.40 \\
\hline Sun et al.2015 & 3.320 & 1.660 & 6.640 & 3.393 & 0.001 & 1.99 & 1.93 \\
\hline Jiang et al.2016 & 4.782 & 1.400 & 16.334 & 2.497 & 0.013 & 0.78 & 1.76 \\
\hline
\end{tabular}

Hazard ratio and $95 \% \mathrm{Cl}$
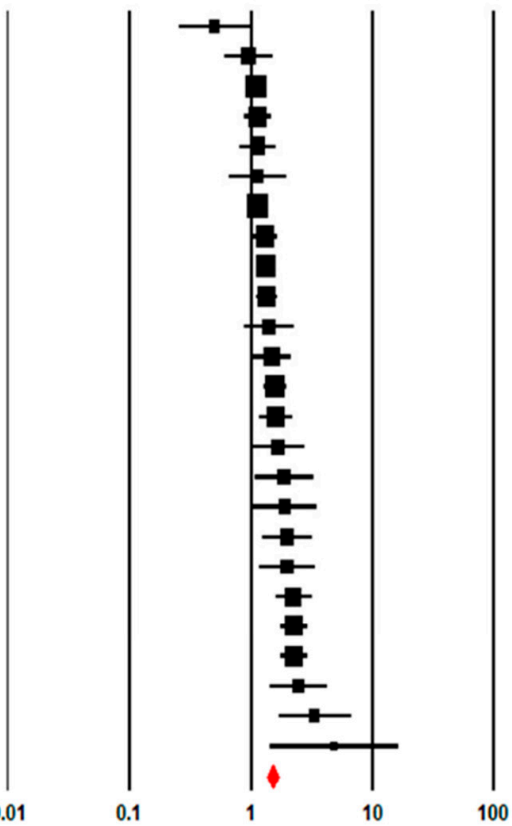

Favours Survival Favours Death

Figure 2. Forest plot of PLR subgroup.

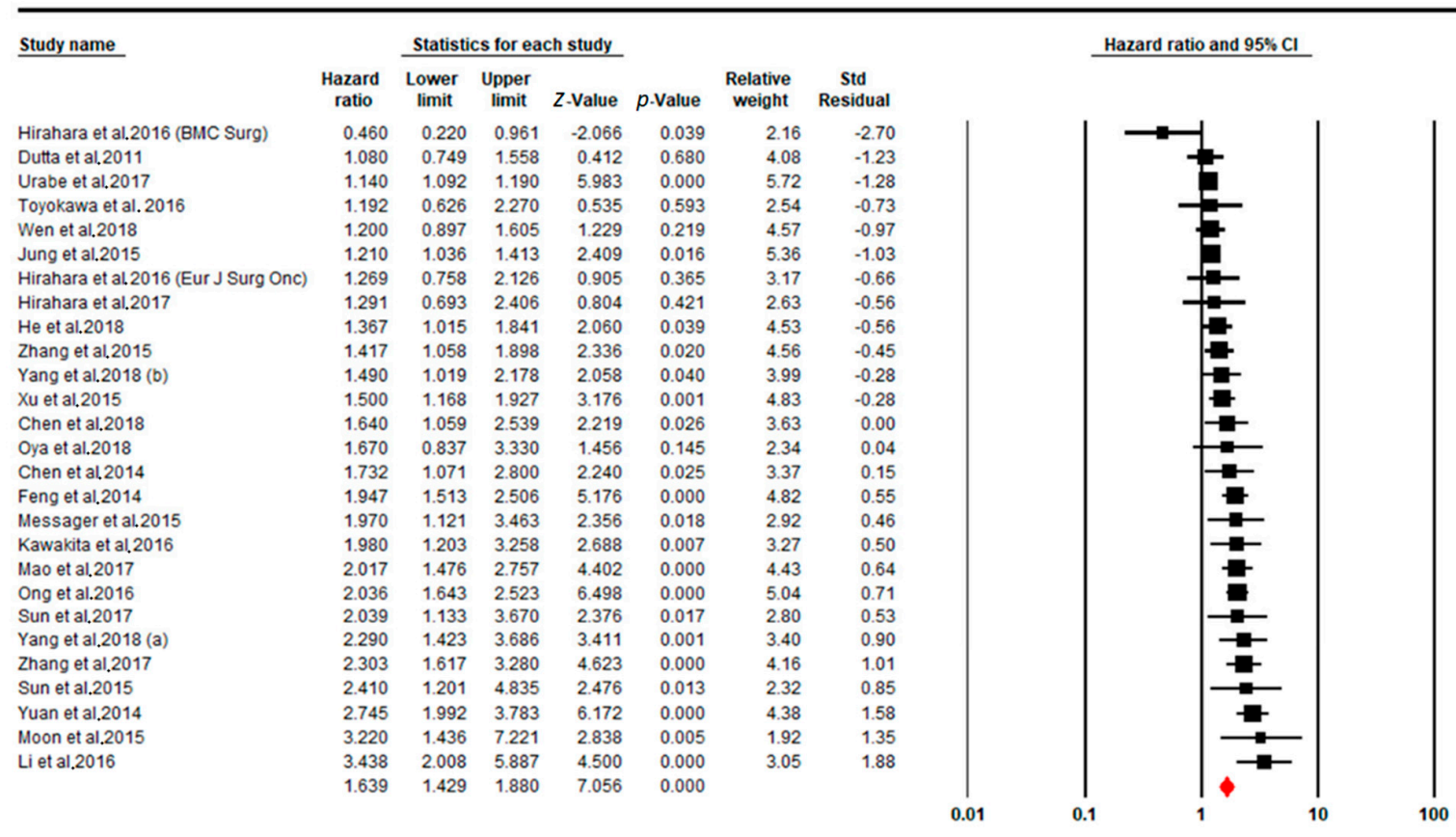

Favours Survival Favours Death

Figure 3. Forest plot of NLR subgroup. 


\subsection{Meta-Analysis MLR Subgroup}

Fewer of the included studies focused on MLR as a prognostic marker, in comparison to PLR and NLR, with a total of only 12 studies being available for inclusion in the assessment of the prognostic efficacy of MLR (Figure 4). In contrast to PLR and NLR, in MLR, the majority of the studies $(n=7)$ showed a negative correlation between increased MLR levels and a worse disease outcome (poorer prognosis), which indicates an increase in patient survival (only 1 study out of the aforementioned 7 had a non-significant effect size, $p<0.05$ ). However, the rest of the included studies $(n=5)$ instead showed a contradictory result, where all five studies indicated a statistically significant effect of MLR levels of poorer patient prognosis $(p<0.05)$. Overall, the pooled effect estimate (HR) was found to not be statistically significant, with a pooled HR value of 1.002 (95\% CI $0.720-1.396 ; p=0.989$ ). Assessment of heterogeneity suggests that there is substantial heterogeneity between the studies included in the subgroup meta-analysis for PLR $\left(\mathrm{I}^{2}=93.902\right.$; $\mathrm{Tau}^{2}=0.292$; Cochran's $Q=180.381)$.

Study name
Furukawa et al.2019
Li et al.2016
Yang et al.2018
Li et al.2013
Ong et al.2016
Urabe et al.2017
Oya et al.2018
Hirahara et al.2016 (Eur J Surg Onc)
Chen et al.2018
Hsu et al.2009
Hirahara et al.2017
Hu et al.2009

\begin{tabular}{|c|c|c|c|c|}
\hline \multirow[b]{2}{*}{$\begin{array}{c}\text { Hazard } \\
\text { ratio }\end{array}$} & \multicolumn{3}{|c|}{ Statistics for each study } & \multirow[b]{2}{*}{$p$-Value } \\
\hline & $\begin{array}{l}\text { Lower } \\
\text { limit }\end{array}$ & $\begin{array}{l}\text { Upper } \\
\text { limit }\end{array}$ & $z$-Value & \\
\hline 0.170 & 0.057 & 0.505 & -3.188 & 0.001 \\
\hline 0.327 & 0.191 & 0.559 & -4.080 & 0.000 \\
\hline 0.550 & 0.372 & 0.814 & -2.991 & 0.003 \\
\hline 0.558 & 0.417 & 0.747 & -3.914 & 0.000 \\
\hline 0.670 & 0.548 & 0.819 & -3.907 & 0.000 \\
\hline 0.830 & 0.774 & 0.890 & -5.232 & 0.000 \\
\hline 0.900 & 0.634 & 1.278 & -0.589 & 0.556 \\
\hline 1.939 & 1.174 & 3.201 & 2.589 & 0.010 \\
\hline 2.020 & 1.295 & 3.152 & 3.098 & 0.002 \\
\hline 2.230 & 1.740 & 2.857 & 6.342 & 0.000 \\
\hline 2.279 & 1.259 & 4.126 & 2.720 & 0.007 \\
\hline 2.970 & 2.099 & 4.202 & 6.148 & 0.000 \\
\hline 1.002 & 0.720 & 1.396 & 0.014 & 0.989 \\
\hline
\end{tabular}

$\begin{array}{rr}\begin{array}{r}\text { Relative } \\ \text { weight }\end{array} & \begin{array}{c}\text { Std } \\ \text { Residual }\end{array} \\ 4.75 & -2.35 \\ 7.77 & -1.93 \\ 8.60 & -1.09 \\ 9.08 & -1.10 \\ 9.43 & -0.77 \\ 9.73 & -0.37 \\ 8.81 & -0.20 \\ 7.98 & 1.15 \\ 8.31 & 1.25 \\ 9.27 & 1.51 \\ 7.44 & 1.38 \\ 8.83 & 2.00 \\ & \end{array}$

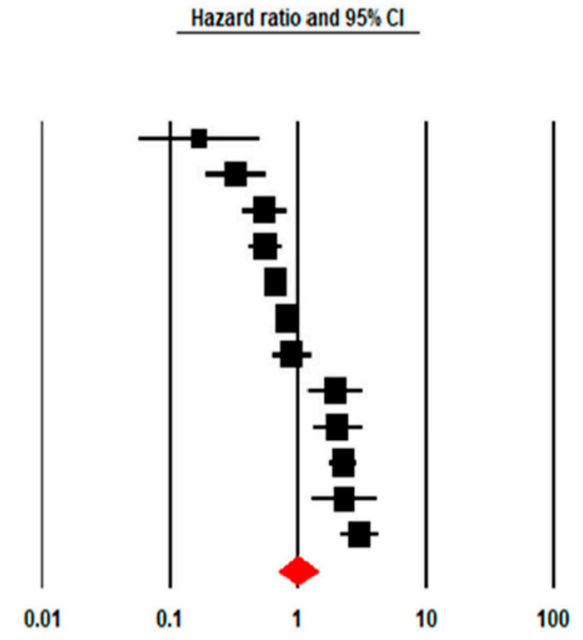

Favours Survival Favours Death

Figure 4. Forest plot of MLR subgroup.

\subsection{Publication Bias}

The Eggers' graphical test was used to assess possible publication bias. The funnel plot was constructed using the same software used to conduct the meta-analysis, CMA (Ver 3.3.070; Biostat, Englewood, NJ, USA). The funnel plots are visualized in Figures (X), (Y) and $(Z)$ for the subgroups, PLR, NLR and MLR, respectively. Orwin's fail-safe N test was applied the publication bias assessment for the PLR and NLR subgroups, for the imputation of multiple missing studies. The funnel plot indicates the presence of publication bias in the PLR and NLR subgroups, which is observed in the significant skew in the distribution of studies along the line of mean effect. However, no significant publication bias was observed in the MLR subgroup.

\subsection{Quality Assessment}

The Newcastle-Ottawa scale (NOS) was used to assess the quality of all included studies. All included studies were found to have quality greater than 2 stars on the assessment scale, which was deemed to be satisfactory for the inclusion of said studies in the meta-analysis. Regardless, the main requirement of inclusion in the study was the availability of good-quality, extractable statistical data from each individual study. 


\section{Discussion}

The current systematic review and meta-analysis were conducted to scrutinize and explore the prognostic potential of PLR, NLR and MLR ratios as prognostic markers in cancer. The use of these prognostic markers has progressed due to the existing established evidence that inflammation can drive cancer growth and progression [3]. In particular, the ease of accessibility, low cost and patient comfort associated with this analysis provide significant benefits if PLR, NLR and MLR are established as clinically reliable cancer prognosticators. Earlier patient cohort studies have investigated the possibility of the use of PLR, NLR and MLR as prognostic markers in cancer [21,22]. However, no comprehensive systematic review or meta-analysis currently exists detailing the comparative efficacy between the three prognostic markers.

A previously published meta-analysis study focused on NLR as a cancer prognostic marker in solid tumors, but did not explore or investigate MLR and PLR as potential prognostic markers in head and neck cancers [23]. Furthermore, while other meta-analysis studies have discussed the efficacy of PLR, NLR and MLR as inflammatory indicators in other chronic conditions, such as infectious diseases and psychosis, their effect in cancer still requires further exploration. While this study looked only at these parameters as predictors of overall outcomes, there remains the possibility that dynamic changes in these parameters during treatment may predict response to treatment. This is especially useful in a tumor such as $\mathrm{HNC}$, especially human papilloma virus (HPV)-negative $\mathrm{HNC}$, because there is currently no universally approved non-invasive and affordable biomarker that serves as a surrogate for tumor burden or treatment response. For HPV-positive cancers, serum levels of HPV DNA show promise as reliable surrogates for treatment response and tumor burden.

This systematic review and meta-analysis study that assessed 49 studies, across nine countries and involving 20,729 patients, investigated the effectiveness of peripheral blood PLR, NLR and MLR ratios in head and neck cancer prognosis. All three of the aforementioned prognostic markers were assessed separately during the meta-analysis as three individual subgroups. It was observed that, overall, more studies had evaluated the prognostic potential of NLR and PLR on patient survival, compared to MLR. The systematic review and meta-analysis study by Mellor et al. serves to validate the findings of this study with regard to PLR as a prognostic marker. While Mellor et al.'s study had a much smaller sample size of included studies $(n=5)$, for assessing patient OS when compared to this study $(n=25)$, the results obtained corroborate and lay a foundation for the findings garnered by Mellor et al., where an increase in PLR levels was found to be significantly associated with poor survival [23]. Furthermore, in the meta-analysis study conducted by Zhu et al., they assessed the effectiveness of PLR and NLR as prognostic markers in ovarian cancer [24]. Despite the difference in the types of cancer being investigated, with the current study assessing the prognostic potential of PLR and NLR in HNC and Zhu et al.'s study investigating ovarian cancer, the results of these twin studies indicated poor OS in cancer patients with higher PLR and NLR levels. A study similar to Zhu et al.'s was conducted by Zhang et al., wherein they focused on highlighting the prognostic potential of PLR and NLR in colorectal cancer. Zhang et al.'s study also reached a similar conclusion, with elevated NLR levels being indicative of poor overall survival in their study [21]. Previous studies, such as the systematic review and meta-analysis by Tham et al., indicate that MLR, unlike PLR and NLR, may have a positive prognostic effect, with elevated MLR levels being correlated with an improved OS [93]. The results of another study by Kano et al. also reflect this statement [88]. However, it is important to note that Tham et al.'s study had a small sample size, pooling five studies for OS, while Kano et al.'s study was a singular retrospective study. Interestingly, the studies used by Tham et al. overlap with the seven studies in our meta-analysis, where we observed a similar pattern of MLR leading to an improved OS. However, when considering the larger sample size of our meta-analysis for MLR, with a near equal number of studies $(n=5)$ showing a statistically significant 
negative effect of increased MLR levels on patient survival, MLR's overall effect on OS remains inconclusive.

This study does have a few limitations that warrant being highlighted. The limited quantity of homogeneous, high-quality literature published in this field impeded detailed subgroup analysis based on clinicopathological and demographic criteria. Additionally, not all studies reported the HR and 95\% CI values in a numerical form, which required $\mathrm{HR}$ and 95\% CI values to be extracted from Kaplan-Meier curves for OS presented in these publications. As extracting numerical data from graphical representations involves estimation, there may be some degree of error introduced into the study, which must be considered when applying the results of this study in the clinical sphere.

Overall, this systematic review and meta-analysis study is validated by previous results seen in other studies in the published literature, while simultaneously bringing into question the results of other studies, by building upon them with a much broader scope of approach and a larger pool of literature incorporated into the meta-analysis. Therefore, the results presented in this paper provide evidence to suggest that PLR and NLR ratios may be capable of being used clinically, as prognostic markers in HNCs, and could, in conjunction with the traditional prognostic indicators of cancer, provide a more robust clinical analysis of patient survival and prognosis in HNC. With regard to MLR, however, there appears to be some contention on its effect on survival in HNC, indicating that further research is needed before a conclusive statement on its clinical utility as a prognostic marker in HNC is presented.

\section{Conclusions}

The study indicates that while PLR and NLR ratios have evidenced potential as prognostic markers for clinical use, particularly in HNC, MLR cannot be currently recommended for clinical use as a prognostic marker. Furthermore, we would like to highlight that the results presented here were obtained from the pooling of multiple individual studies, which each had their own study design, parameters and analysis methods. Therefore, despite the results presented here and previous studies validating these results, the inherent heterogeneity between individual studies being pooled requires that further large-scale clinical studies with a large sample size and a homogeneous approach are conducted before bringing these prognostic markers into clinical practice. Therefore, large-scale, longitudinal patient studies focusing on PLR and NLR as prognostic markers are necessary before they can be incorporated into standard practice as complementary biomarkers to currently existing prognostic markers in cancer. Until then, the results of this systematic review and meta-analysis serve to aid in both clinical decision-making and ongoing and future research in this field.

Supplementary Materials: The following are available online at https:/ /www.mdpi.com/article/10.3 390/cancers13164166/s1, Table S1: PRISMA checklist for systematic reviews and meta-analyses detailing the compliance of study with PRISMA method.

Author Contributions: Conceptualization, C.K. and R.J.; Methodology, C.K.; Software R.J.; Validation, K.S., D.S., S.S., G.K.M., S.B.; Formal Analysis, C.K., R.J.; Investigation, C.K., V.T.; Resources, V.T.; Data Curation, C.K., V.T.; writing — original draft preparation, C.K.; writing—review and editing, K.S., D.S., S.S., G.K.M., S.B.; visualization, R.J.; Supervision, R.J.; project administration, R.J. All authors have read and agreed to the published version of the manuscript.

Funding: This work was not supported by any external funding.

Acknowledgments: All contributors have been acknowledged in authorship.

Conflicts of Interest: All authors involved in the manuscript declare no conflict of interest. 


\section{References}

1. Wang, M.; Zhao, J.; Zhang, L.; Wei, F.; Lian, Y.; Wu, Y.; Gong, Z.; Zhang, S.; Zhou, J.; Cao, K. Role of tumor microenvironment in tumorigenesis. J. Cancer 2017, 8, 761. [CrossRef]

2. Colotta, F.; Allavena, P.; Sica, A.; Garlanda, C.; Mantovani, A. Cancer-related inflammation, the seventh hallmark of cancer: Links to genetic instability. Carcinogenesis 2009, 30, 1073-1081. [CrossRef] [PubMed]

3. Coussens, L.M.; Werb, Z. Inflammation and cancer. Nature 2002, 420, 860-867. [CrossRef] [PubMed]

4. Gao, Y.; Wang, W.J.; Zhi, Q.; Shen, M.; Jiang, M.; Bian, X.; Gong, F.R.; Zhou, C.; Lian, L.; Wu, M.Y.; et al. Neutrophil/lymphocyte ratio is a more sensitive systemic inflammatory response biomarker than platelet/lymphocyte ratio in the prognosis evaluation of unresectable pancreatic cancer. Oncotarget 2017, 8, 88835-88844. [CrossRef] [PubMed]

5. Akinci Ozyurek, B.; Sahin Ozdemirel, T.; Buyukyaylaci Ozden, S.; Erdogan, Y.; Kaplan, B.; Kaplan, T. Prognostic Value of the Neutrophil to Lymphocyte Ratio (NLR) in Lung Cancer Cases. Asian Pac. J. Cancer Prev. 2017, 18, 1417-1421. [CrossRef] [PubMed]

6. Krenn-Pilko, S.; Langsenlehner, U.; Thurner, E.M.; Stojakovic, T.; Pichler, M.; Gerger, A.; Kapp, K.S.; Langsenlehner, T. The elevated preoperative platelet-to-lymphocyte ratio predicts poor prognosis in breast cancer patients. Br. J. Cancer 2014, $110,2524$. [CrossRef]

7. Yan, M.; Jurasz, P. The role of platelets in the tumor microenvironment: From solid tumors to leukemia. Biochim. Biophys. Acta BBA Mol. Cell Res. 2016, 1863, 392-400. [CrossRef]

8. Shi, C.; Pamer, E.G. Monocyte recruitment during infection and inflammation. Nat. Reviews. Immunol. 2011, 11, 762-774. [CrossRef]

9. McMillan, D.C. An inflammation-based prognostic score and its role in the nutrition-based management of patients with cancer: Nutrition Society and BAPEN Medical Symposium on 'Nutrition support in cancer therapy'. Proc. Nutr. Soc. 2008, 67, 257-262. [CrossRef]

10. Fearon, K.C.H. Cancer cachexia: Developing multimodal therapy for a multidimensional problem. Eur. J. Cancer 2008, 44, 1124-1132. [CrossRef]

11. Pardoll, D.M.; Topalian, S.L. The role of CD4+ T cell responses in antitumor immunity. Curr. Opin. Immunol. 1998, 10, 588-594. [CrossRef]

12. Hung, K.; Hayashi, R.; Lafond-Walker, A.; Lowenstein, C.; Pardoll, D.; Levitsky, H. The central role of CD4+ T cells in the antitumor immune response. J. Exp. Med. 1998, 188, 2357-2368. [CrossRef]

13. Chanmee, T.; Ontong, P.; Konno, K.; Itano, N. Tumor-associated macrophages as major players in the tumor microenvironment. Cancers 2014, 6, 1670-1690. [CrossRef] [PubMed]

14. Shi, L.; Qin, X.; Wang, H.; Xia, Y.; Li, Y.; Chen, X.; Shang, L.; Tai, Y.T.; Feng, X.; Acharya, P.; et al. Elevated neutrophilto-lymphocyte ratio and monocyte-to-lymphocyte ratio and decreased platelet-to-lymphocyte ratio are associated with poor prognosis in multiple myeloma. Oncotarget 2017, 8, 18792-18801. [CrossRef]

15. Sun, X.; Cui, F.; Yin, H.; Wu, D.; Wang, N.; Yuan, M.; Fei, Y.; Wang, Q. Association between EGFR mutation and expression of BRCA1 and RAP80 in non-small cell lung cancer. Oncol. Lett. 2018, 16, 2201-2206. [CrossRef]

16. Rosen, E.M.; Fan, S.; Pestell, R.G.; Goldberg, I.D. BRCA1 gene in breast cancer. J. Cell. Physiol. 2003, 196, 19-41. [CrossRef] [PubMed]

17. Brennan, D.J.; Gallagher, W.M. Prognostic ability of a panel of immunohistochemistry markers-retailoring of an 'old solution'. Breast Cancer Res. BCR 2008, 10, 102. [CrossRef] [PubMed]

18. Mocellin, S.; Rossi, C.R.; Pilati, P.; Nitti, D.; Marincola, F.M. Quantitative real-time PCR: A powerful ally in cancer research. Trends Mol. Med. 2003, 9, 189-195. [CrossRef]

19. Ying, H.-Q.; Deng, Q.-W.; He, B.-S.; Pan, Y.-Q.; Wang, F.; Sun, H.-L.; Chen, J.; Liu, X.; Wang, S.-K. The prognostic value of preoperative NLR, d-NLR, PLR and LMR for predicting clinical outcome in surgical colorectal cancer patients. Med Oncol. 2014, 31, 305. [CrossRef]

20. Chen, L.; Zeng, H.; Yang, J.; Lu, Y.; Zhang, D.; Wang, J.; Kuang, C.; Zhu, S.; Wang, M.; Ma, X. Survival and prognostic analysis of preoperative inflammatory markers in patients undergoing surgical resection for laryngeal squamous cell carcinoma. $B M C$ Cancer 2018, 18, 816. [CrossRef]

21. Zhang, J.; Zhang, H.Y.; Li, J.; Shao, X.Y.; Zhang, C.X. The elevated NLR, PLR and PLT may predict the prognosis of patients with colorectal cancer: A systematic review and meta-analysis. Oncotarget 2017, 8, 68837-68846. [CrossRef]

22. Solmaz, S.; Uzun, O.; Acar, C.; Sevindik, O.G.; Piskin, O.; Ozsan, H.G.; Demirkan, F.; Undar, B.; Alacacioglu, A.; Ozcan, M.A. Is the platelet-to-lymphocyte ratio a new prognostic marker in multiple myeloma? J. Lab. Physicians 2018, 10, 363. [CrossRef]

23. Mellor, K.L.; Powell, A.G.M.T.; Lewis, W.G. Systematic Review and Meta-Analysis of the Prognostic Significance of NeutrophilLymphocyte Ratio (NLR) After R0 Gastrectomy for Cancer. J. Gastrointest. Cancer 2018, 49, 237-244. [CrossRef] [PubMed]

24. Zhu, Y.; Zhou, S.; Liu, Y.; Zhai, L.; Sun, X. Prognostic value of systemic inflammatory markers in ovarian Cancer: A PRISMAcompliant meta-analysis and systematic review. BMC Cancer 2018, 18, 443. [CrossRef] [PubMed]

25. Moher, D.; Shamseer, L.; Clarke, M.; Ghersi, D.; Liberati, A.; Petticrew, M.; Shekelle, P.; Stewart, L.A. Preferred reporting items for systematic review and meta-analysis protocols (PRISMA-P) 2015 statement. Syst. Rev. 2015, 4, 1. [CrossRef]

26. Kumarasamy, C.; Sabarimurugan, S.; Madurantakam, R.M.; Lakhotiya, K.; Samiappan, S.; Baxi, S.; Nachimuthu, R.; Gothandam, K.M.; Jayaraj, R. Prognostic significance of blood inflammatory biomarkers NLR, PLR, and LMR in cancer-A protocol for systematic review and meta-analysis. Medicine 2019, 98, e14834. [CrossRef] [PubMed] 
27. Wells, G.A.; Shea, B.; O'connell, D.; Peterson, J.; Welch, V.; Losos, M.; Tugwell, P. The Newcastle-Ottawa Scale (NOS) for Assessing the Quality of Nonrandomised Studies in Meta-Analyses; Ottawa Hospital Research Institute: Ottawa, ON, Canada, 2009.

28. Jayaraj, R.; Kumarasamy, C.; Madhav, M.R.; Pandey, V.; Sabarimurugan, S.; Ramesh, N.; Gothandam, K.M.; Baxi, S. Comment on "Systematic Review and Meta-Analysis of Diagnostic Accuracy of miRNAs in Patients with Pancreatic Cancer". Dis. Markers 2018, 2018, 2. [CrossRef]

29. Jayaraj, R.; Kumarasamy, C. Comment on 'Prognostic biomarkers for oral tongue squamous cell carcinoma: A systematic review and meta-analysis'. Br. J. Cancer 2018, 118, e11. [CrossRef] [PubMed]

30. Jayaraj, R.; Kumarasamy, C.J.O.O. Comment on, "Survival for HPV-positive oropharyngeal squamous cell carcinoma with surgical versus non-surgical treatment approach: A systematic review and meta-analysis". Oral. Oncol. 2018, 90, 137-138. [CrossRef]

31. Jayaraj, R.; Kumarasamy, C.; Sabarimurugan, S.; Baxi, S.J.F.i.P. Commentary: Blood-Derived microRNAs for Pancreatic Cancer Diagnosis: A Narrative Review and Meta-Analysis. Front. Physiol. 2018, 9, 1896. [CrossRef]

32. Jayaraj, R.; Kumarasamy, C.J.O.O. Conceptual interpretation of analysing and reporting of results on systematic review and meta-analysis of optimal extent of lateral neck dissection for well-differentiated thyroid carcinoma with metastatic lateral neck lymph nodes. Oral. Oncol. 2019, 89, 153-154. [CrossRef]

33. Borenstein, M.; Rothstein, H. Comprehensive Meta-Analysis; Biostat, 1999. Available online: https://www.meta-analysis.com/ downloads/Meta-Analysis\%20Manual\%20V3.pdf (accessed on 17 August 2021).

34. Borenstein, M.; Hedges, L.V.; Higgins, J.P.T.; Rothstein, H.R. A basic introduction to fixed-effect and random-effects models for meta-analysis. Res. Synth. Methods 2010, 1, 97-111. [CrossRef]

35. Jayaraj, R.; Kumarasamy, C.; Gothandam, K.M. Letter to the editor "Prognostic value of microRNAs in colorectal cancer: A meta-analysis". Cancer Manag. Res. 2018, 10, 3501-3503. [CrossRef] [PubMed]

36. Jayaraj, R.; Kumarasamy, C.J.O.o. Letter to the Editor about the Article: "Performance of different imaging techniques in the diagnosis of head and neck cancer mandibular invasion: A systematic review and meta-analysis". Oral. Oncol. 2018, 89, 159-160. [CrossRef]

37. Jayaraj, R.; Kumarasamy, C.; Sabarimurugan, S.; Baxi, S. Letter to the Editor in response to the article," The epidemiology of oral human papillomavirus infection in healthy populations: A systematic review and meta-analysis". Oral. Oncol. 2018, 84, 121-122. [CrossRef] [PubMed]

38. Jayaraj, R.; Kumarasamy, C.; Samiappan, S.; Swaminathan, P. Letter to the Editor regarding, "The prognostic role of PD-L1 expression for survival in head and neck squamous cell carcinoma: A systematic review and meta-analysis". Oral. Oncol. 2018. [CrossRef] [PubMed]

39. Jayaraj, R.; Kumarasamy, C.; Madurantakam Royam, M.; Devi, A.; Baxi, S. Letter to the editor: Is HIF-1 $\alpha$ a viable prognostic indicator in OSCC? A critical review of a meta-analysis study. World J. Surg. Oncol. 2018, 16, 111. [CrossRef] [PubMed]

40. Higgins, J.P.T.; Thompson, S.G.; Deeks, J.J.; Altman, D.G. Measuring inconsistency in meta-analyses. BMJ Br. Med. J. 2003, 327, 557. [CrossRef]

41. von Hippel, P.T. The heterogeneity statistic I (2) can be biased in small meta-analyses. BMC Med. Res. Methodol. 2015, 15, 35. [CrossRef] [PubMed]

42. Huedo-Medina, T.B.; Sánchez-Meca, J.; Marín-Martínez, F.; Botella, J. Assessing heterogeneity in meta-analysis: Q statistic or I ${ }^{2}$ index? Psychol. Methods 2006, 11, 193. [CrossRef] [PubMed]

43. Deeks, J.J.; Higgins, J.P.T.; Altman, D.G. Analysing data and undertaking meta-analyses. In Cochrane Handbook for Systematic Reviews of Interventions; Cochrane Book Series; Wiley: Hoboken, NJ, USA, 2008; pp. 243-296.

44. Egger, M.; Smith, G.D.; Schneider, M.; Minder, C. Bias in meta-analysis detected by a simple, graphical test. BMJ 1997, 315, 629-634. [CrossRef]

45. Orwin, R.G. A fail-safe N for effect size in meta-analysis. J. Educ. Stat. 1983, 8, 157-159. [CrossRef]

46. Begg, C.B.; Mazumdar, M. Operating characteristics of a rank correlation test for publication bias. Biometrics 1994, 50, $1088-1101$. [CrossRef] [PubMed]

47. Wen, J.; Bedford, M.; Begum, R.; Mitchell, H.; Hodson, J.; Whiting, J.; Griffiths, E. The value of inflammation based prognostic scores in patients undergoing surgical resection for oesophageal and gastric carcinoma. J. Surg. Oncol. 2018, 117, $1697-1707$. [CrossRef] [PubMed]

48. Kawakita, D.; Tada, Y.; Imanishi, Y.; Beppu, S.; Tsukahara, K.; Kano, S.; Ozawa, H.; Okami, K.; Sato, Y.; Shimizu, A. Impact of hematological inflammatory markers on clinical outcome in patients with salivary duct carcinoma: A multi-institutional study in Japan. Oncotarget 2017, 8, 1083. [CrossRef] [PubMed]

49. Chen, C.; Sun, P.; Dai, Q.-s.; Weng, H.-w.; Li, H.-p.; Ye, S. The Glasgow Prognostic Score predicts poor survival in cisplatin-based treated patients with metastatic nasopharyngeal carcinoma. PLoS ONE 2014, 9, e112581. [CrossRef]

50. Zhang, F.; Chen, Z.; Wang, P.; Hu, X.; Gao, Y.; He, J. Combination of platelet count and mean platelet volume (COP-MPV) predicts postoperative prognosis in both resectable early and advanced stage esophageal squamous cell cancer patients. Tumor Biol. 2016, 37, 9323-9331. [CrossRef]

51. Jiang, K.; Lei, J.; Li, C.; Shu, K.; Li, W.; Zhang, Y.; Li, Z.; Gong, R.; Zhu, J. Comparison of the prognostic values of selected inflammation based scores in patients with medullary thyroid carcinoma: A pilot study. J. Surg. Oncol. 2017, 116, 281-287. [CrossRef] 
52. Li, X.H.; Chang, H.; Xu, B.Q.; Tao, Y.L.; Gao, J.; Chen, C.; Qu, C.; Zhou, S.; Liu, S.R.; Wang, X.H. An inflammatory biomarker-based nomogram to predict prognosis of patients with nasopharyngeal carcinoma: An analysis of a prospective study. Cancer Med. 2017, 6, 310-319. [CrossRef]

53. Feng, J.-F.; Huang, Y.; Chen, Q.-X. Preoperative platelet lymphocyte ratio (PLR) is superior to neutrophil lymphocyte ratio (NLR) as a predictive factor in patients with esophageal squamous cell carcinoma. World J. Surg. Oncol. 2014, 12, 1-6. [CrossRef]

54. Jiang, R.; Zou, X.; Hu, W.; Fan, Y.-Y.; Yan, Y.; Zhang, M.-X.; You, R.; Sun, R.; Luo, D.-H.; Chen, Q.-Y. The elevated pretreatment platelet-to-lymphocyte ratio predicts poor outcome in nasopharyngeal carcinoma patients. Tumor Biol. 2015, 36, 7775-7787. [CrossRef] [PubMed]

55. Jung, J.; Park, S.Y.; Park, S.-j.; Park, J. Prognostic value of the neutrophil-to-lymphocyte ratio for overall and disease-free survival in patients with surgically treated esophageal squamous cell carcinoma. Tumor Biol. 2016, 37, 7149-7154. [CrossRef] [PubMed]

56. He, Y.F.; Luo, H.Q.; Wang, W.; Chen, J.; Yao, Y.W.; Yan, Y.; Wu, S.S.; Hu, X.X.; Ke, L.H.; Niu, J.Y. Preoperative NLR and PLR in the middle or lower ESCC patients with radical operation. Eur. J. Cancer Care 2017, 26, e12445. [CrossRef] [PubMed]

57. Jiang, K.; Lei, J.; Chen, W.; Gong, Y.; Luo, H.; Li, Z.; Gong, R.; Zhu, J. Association of the preoperative neutrophil-to-lymphocyte and platelet-to-lymphocyte ratios with lymph node metastasis and recurrence in patients with medullary thyroid carcinoma. Medicine 2016, 95, e5079. [CrossRef] [PubMed]

58. Hirahara, N.; Tajima, Y.; Fujii, Y.; Yamamoto, T.; Hyakudomi, R.; Hirayama, T.; Taniura, T.; Ishitobi, K.; Kidani, A.; Kawabata, Y. A novel prognostic scoring system using inflammatory response biomarkers for esophageal squamous cell carcinoma. World J. Surg. 2018, 42, 172-184. [CrossRef]

59. Ong, H.S.; Gokavarapu, S.; Wang, L.Z.; Tian, Z.; Zhang, C.P. Low pretreatment lymphocyte-monocyte ratio and high plateletlymphocyte ratio indicate poor cancer outcome in early tongue cancer. Int. J. Oral Maxillofac. Surg. 2017, 75, 1762-1774. [CrossRef]

60. Mao, Y.; Fu, Y.; Gao, Y.; Yang, A.; Zhang, Q. Platelet-to-lymphocyte ratio predicts long-term survival in laryngeal cancer. Eur. Arch. Oto-Rhino-Laryngol. 2018, 275, 553-559. [CrossRef]

61. Dutta, S.; Crumley, A.B.C.; Fullarton, G.M.; Horgan, P.G.; McMillan, D.C. Comparison of the prognostic value of tumour and patient related factors in patients undergoing potentially curative resection of gastric cancer. Am. J. Surg. 2012, 204, 294-299. [CrossRef]

62. Li, J.-P.; Chen, S.-L.; Liu, X.-M.; He, X.; Xing, S.; Liu, Y.-J.; Lin, Y.-H.; Liu, W.-L. A novel inflammation-based stage (I Stage) predicts overall survival of patients with nasopharyngeal carcinoma. Int. J. Mol. Sci. 2016, 17, 1900. [CrossRef]

63. Messager, M.; Neofytou, K.; Chaudry, M.A.; Allum, W.H. Prognostic impact of preoperative platelets to lymphocytes ratio (PLR) on survival for oesophageal and junctional carcinoma treated with neoadjuvant chemotherapy: A retrospective monocentric study on 153 patients. Eur. J. Surg. Oncol. 2015, 41, 1316-1323. [CrossRef]

64. Hirahara, N.; Matsubara, T.; Kawahara, D.; Nakada, S.; Ishibashi, S.; Tajima, Y. Prognostic significance of preoperative inflammatory response biomarkers in patients undergoing curative thoracoscopic esophagectomy for esophageal squamous cell carcinoma. Eur. J. Surg. Oncol. 2017, 43, 493-501. [CrossRef] [PubMed]

65. Moon, H.; Roh, J.-L.; Lee, S.-w.; Kim, S.-B.; Choi, S.-H.; Nam, S.Y.; Kim, S.Y. Prognostic value of nutritional and hematologic markers in head and neck squamous cell carcinoma treated by chemoradiotherapy. Radiother. Oncol. 2016, 118, 330-334. [CrossRef]

66. Hirahara, N.; Matsubara, T.; Mizota, Y.; Ishibashi, S.; Tajima, Y. Prognostic value of preoperative inflammatory response biomarkers in patients with esophageal cancer who undergo a curative thoracoscopic esophagectomy. BMC Surg. 2016, 16, 1-12. [CrossRef] [PubMed]

67. Turri-Zanoni, M.; Salzano, G.; Lambertoni, A.; Giovannardi, M.; Karligkiotis, A.; Battaglia, P.; Castelnuovo, P. Prognostic value of pretreatment peripheral blood markers in paranasal sinus cancer: Neutrophil-to-lymphocyte and platelet-to-lymphocyte ratio. Head Neck 2017, 39, 730-736. [CrossRef]

68. Xie, X.; Luo, K.J.; Hu, Y.; Wang, J.Y.; Chen, J. Prognostic value of preoperative platelet-lymphocyte and neutrophil-lymphocyte ratio in patients undergoing surgery for esophageal squamous cell cancer. Dis. Esophagus 2016, 29, 79-85. [CrossRef]

69. Bojaxhiu, B.; Templeton, A.J.; Elicin, O.; Shelan, M.; Zaugg, K.; Walser, M.; Giger, R.; Aebersold, D.M.; Dal Pra, A. Relation of baseline neutrophil-to-lymphocyte ratio to survival and toxicity in head and neck cancer patients treated with (chemo-) radiation. Radiat. Oncol. 2018, 13, 1-9. [CrossRef] [PubMed]

70. Sun, P.; Chen, C.; Xia, Y.; Bi, X.; Liu, P.; Zhang, F.; Yang, H.; An, X.; Jiang, W.; Wang, F. The ratio of C-reactive protein/albumin is a novel inflammatory predictor of overall survival in cisplatin-based treated patients with metastatic nasopharyngeal carcinoma. Dis. Markers 2017, 2017, 6570808. [CrossRef] [PubMed]

71. Sun, W.; Zhang, L.; Luo, M.; Hu, G.; Mei, Q.; Liu, D.; Long, G.; Hu, G. Pretreatment hematologic markers as prognostic factors in patients with nasopharyngeal carcinoma: Neutrophil-lymphocyte ratio and platelet-lymphocyte ratio. Head Neck 2016, 38, E1332-E1340. [CrossRef]

72. Tangthongkum, M.; Tiyanuchit, S.; Kirtsreesakul, V.; Supanimitjaroenporn, P.; Sinkitjaroenchai, W. Platelet to lymphocyte ratio and red cell distribution width as prognostic factors for survival and recurrence in patients with oral cancer. Eur. Arch. Oto-Rhino-Laryngol. 2017, 274, 3985-3992. [CrossRef]

73. Ozturk, K.; Akyildiz, N.S.; Uslu, M.; Gode, S.; Uluoz, U. The effect of preoperative neutrophil, platelet and lymphocyte counts on local recurrence and survival in early-stage tongue cancer. Eur. Arch. Oto-Rhino-Laryngol. 2016, 273, 4425-4429. [CrossRef] 
74. Toyokawa, T.; Kubo, N.; Tamura, T.; Sakurai, K.; Amano, R.; Tanaka, H.; Muguruma, K.; Yashiro, M.; Hirakawa, K.; Ohira, M. The pretreatment Controlling Nutritional Status (CONUT) score is an independent prognostic factor in patients with resectable thoracic esophageal squamous cell carcinoma: Results from a retrospective study. BMC Cancer 2016, 16, 1-11. [CrossRef] [PubMed]

75. Urabe, M.; Yamashita, H.; Watanabe, T.; Seto, Y. Comparison of prognostic abilities among preoperative laboratory data indices in patients with resectable gastric and esophagogastric junction adenocarcinoma. World J. Surg. 2018, 42, 185-194. [CrossRef] [PubMed]

76. Wang, K.-f.; Chang, B.-y.; Chen, X.-q.; Liu, P.-p.; Wuxiao, Z.-j.; Wang, Z.-h.; Li, S.; Jiang, W.-q.; Xia, Z.-j. A prognostic model based on pretreatment platelet lymphocyte ratio for stage IE/IIE upper aerodigestive tract extranodal NK/T cell lymphoma, nasal type. Med. Oncol. 2014, 31, 1-7. [CrossRef] [PubMed]

77. Wei, X.-1.; Wang, F.-h.; Zhang, D.-s.; Qiu, M.-z.; Ren, C.; Jin, Y.; Zhou, Y.-x.; Wang, D.-s.; He, M.-m.; Bai, L. A novel inflammationbased prognostic score in esophageal squamous cell carcinoma: The C-reactive protein/albumin ratio. BMC Cancer 2015, 15, 1-11. [CrossRef]

78. Xu, X.-L.; Yu, H.-Q.; Hu, W.; Song, Q.; Mao, W.-M. A novel inflammation-based prognostic score, the C-reactive protein/albumin ratio predicts the prognosis of patients with operable esophageal squamous cell carcinoma. PLoS ONE 2015, 10, e0138657. [CrossRef]

79. Yang, Y.; Xu, H.; Zhou, L.; Deng, T.; Ning, T.; Liu, R.; Zhang, L.; Wang, X.; Ge, S.; Li, H. Platelet to lymphocyte ratio is a predictive marker of prognosis and therapeutic effect of postoperative chemotherapy in non-metastatic esophageal squamous cell carcinoma. Clin. Chim. Acta 2018, 479, 160-165. [CrossRef]

80. Ye, L.; Oei, R.W.; Kong, F.; Xu, T.; Shen, C.; Wang, X.; He, X.; Kong, L.; Hu, C.; Ying, H. Prognostic values of hematological biomarkers in nasopharyngeal carcinoma patients treated with intensity-modulated radiotherapy. Eur. Arch. Oto-Rhino-Laryngol. 2018, 275, 1309-1317. [CrossRef]

81. Yuan, D.; Zhu, K.; Li, K.; Yan, R.; Jia, Y.; Dang, C. The preoperative neutrophil-lymphocyte ratio predicts recurrence and survival among patients undergoing R0 resections of adenocarcinomas of the esophagogastric junction. J. Surg. Oncol. 2014, 110, 333-340. [CrossRef] [PubMed]

82. Zhang, L.; Su, Y.; Chen, Z.; Wei, Z.; Han, W.; Xu, A. The prognostic value of preoperative inflammation-based prognostic scores and nutritional status for overall survival in resected patients with nonmetastatic Siewert type II/III adenocarcinoma of esophagogastric junction. Medicine 2017, 96, e7647. [CrossRef] [PubMed]

83. Hsu, W.H.; Hsu, P.K.; Hsieh, C.C.; Huang, C.S.; Wu, Y.C. The metastatic lymph node number and ratio are independent prognostic factors in esophageal cancer. J. Gastrointest. Surg. 2009, 13, 1913-1920. [CrossRef]

84. Chien, H.-C.; Chen, H.-S.; Wu, S.-C.; Hsu, P.-K.; Liu, C.-Y.; Wang, B.-Y.; Shih, C.-H.; Liu, C.-C. The prognostic value of metastatic lymph node number and ratio in oesophageal squamous cell carcinoma patients with or without neoadjuvant chemoradiation. Eur. J. Cardiothorac. Surg. 2016, 50, 337-343. [CrossRef]

85. Furukawa, K.; Kawasaki, G.; Naruse, T.; Umeda, M. Prognostic Significance of Pretreatment Lymphocyte-to-Monocyte Ratio in Patients with Tongue Cancer. Anticancer Res. 2019, 39, 405. [CrossRef] [PubMed]

86. Hsueh, C.; Tao, L.; Zhang, M.; Cao, W.; Gong, H.; Zhou, J.; Zhou, L. The prognostic value of preoperative neutrophils, platelets, lymphocytes, monocytes and calculated ratios in patients with laryngeal squamous cell cancer. Oncotarget 2017, 8, 60514-60527. [CrossRef]

87. Huang, Y.; Feng, J.-F. Low preoperative lymphocyte to monocyte ratio predicts poor cancer-specific survival in patients with esophageal squamous cell carcinoma. OncoTargets Ther. 2015, 8, 137-145.

88. Kano, S.; Homma, A.; Hatakeyama, H.; Mizumachi, T.; Sakashita, T.; Kakizaki, T.; Fukuda, S. Pretreatment lymphocyte-tomonocyte ratio as an independent prognostic factor for head and neck cancer. Head Neck 2017, 39, 247-253. [CrossRef]

89. Li, J.; Jiang, R.; Liu, W.-S.; Liu, Q.; Xu, M.; Feng, Q.-S.; Chen, L.-Z.; Bei, J.-X.; Chen, M.-Y.; Zeng, Y.-X. A Large Cohort Study Reveals the Association of Elevated Peripheral Blood Lymphocyte-to-Monocyte Ratio with Favorable Prognosis in Nasopharyngeal Carcinoma. PLoS ONE 2013, 8, e83069. [CrossRef] [PubMed]

90. Liu, J.-S.; Huang, Y.; Yang, X.; Feng, J.-F. A nomogram to predict prognostic values of various inflammatory biomarkers in patients with esophageal squamous cell carcinoma. Am. J. Cancer Res. 2015, 5, 2180-2189.

91. Oya, R.; Takenaka, Y.; Aoki, K.; Hamaguchi, H.; Takemura, K.; Nozawa, M.; Kitamura, T.; Yamamoto, Y.; Uno, A. Prognostic Significance of Hematologic Markers in Patients with Head and Neck Squamous Cell Carcinomas. Int. J. Otorhinolaryngol. Head Neck Surg. 2018, 7, 55-65. [CrossRef]

92. Yang, J.; Hsueh, C.-Y.; Cao, W.; Zhou, L. Pretreatment lymphocyte-to-monocyte ratio as an independent prognostic factor for hypopharyngeal squamous cell carcinoma. Acta Otolaryngol. 2018, 138, 734-740. [CrossRef] [PubMed]

93. Tham, T.; Olson, C.; Khaymovich, J.; Herman, S.W.; Costantino, P.D. The lymphocyte-to-monocyte ratio as a prognostic indicator in head and neck cancer: A systematic review and meta-analysis. Eur. Arch. Oto-Rhino-Laryngol. 2018, 275, 1663-1670. [CrossRef] 